\title{
The Haroche-Ramsey experiment as a generalized measurement
}

\author{
W.M. de Muynck and A.J.A. Hendrikx \\ Theoretical Physics, Eindhoven University of Technology, \\ P.O.B. 513, 5600 MB Eindhoven, The Netherlands
}

(November 14, 2018)

\begin{abstract}
A number of atomic beam experiments, related to the Ramsey experiment and a recent experiment by Brune et al. [价, are studied with respect to the question of complementarity. Three different procedures for obtaining information on the state of the incoming atom are compared. Positive operator-valued measures are explicitly calculated. It is demonstrated that, in principle, it is possible to choose the experimental arrangement so as to admit an interpretation as a joint non-ideal measurement yielding interference and "which-way" information. Comparison of the different measurements gives insight into the question of which information is provided by a (generalized) quantum mechanical measurement. For this purpose the subspaces of Hilbert-Schmidt space, spanned by the operators of the POVM, are determined for different measurement arrangements and different values of the parameters.

Pacs number(s): 03.65.Bz, 03.65.Ca
\end{abstract}

\section{INTRODUCTION}

In the standard formalism of quantum mechanics a measurement corresponds to a self-adjoint operator yielding its eigenvalues as measurement results, with probabilities determined by the expectation values of the projections on its eigenvectors. It is by now well-known [2] that this formalism is too restricted to encompass all possible experiments within the domain of application of quantum mechanics. Many experiments performed in actual practice are of the type of generalized measurements yielding probabilities determined by the expectation values of so-called positive operator-valued measures (POVMs) rather than projection-valued ones. The theory describing this generalization is referred to as the operational approach.

Such generalized measurements are important for two different reasons. First, from a fundamental point of view they are interesting because they enable to transcend the bounds imposed by the standard formalism on the notion of a quantum mechanical measurement. In particular, the projection postulate does not make sense in a generalized measurement because in general no projection operators are available. The inapplicability of this postulate in most realistic measurement procedures indeed demonstrates the restricted scope of the standard formalism. What is a quantum mechanical measurement, and which observable is measured by a particular measurement procedure must be determined by considering in detail the interaction of the microscopic object and the measuring instrument as a quantum mechanical process. In the second place generalized measurements are interesting from a practical point of view because they can yield more information on the state of the object than is provided by the measurement of a standard observable.

We should distinguish between the determinative and the preparative aspects of measurement [3]. The former is related to the final state of the measuring instrument, and the information on the initial object state that is provided by the measurement. The latter refers to the preparation of the post-measurement state of the object. Unfortunately, these different aspects of measurement have been confounded ever since in the Copenhagen interpretation a measurement has been defined as a preparation of the object in a final state described by an eigenvector of the measured observable. It is important to note that most measurement procedures do not satisfy this criterion. Due to the ensuing confusion even recently the role of the Heisenberg uncertainty relations in quantum measurement has been a source of controversy [4] 6]. Whereas Storey et al. 泊 conclude that "the principle of complementarity is a consequence of the Heisenberg uncertainty relation," Scully et al. [5] observe that "The principle of complementarity is manifest although the positionmomentum uncertainty relation plays no role." Dürr et al. [6] stress that quantum correlations due to the interaction of object and detector, rather than "classical" momentum transfer, enforces the loss of interference in a 'which-way' measurement. In their experiment momentum disturbance is not large enough to account for the loss of interference if the measurement arrangement is changed so as to yield 'which-way' information. These diverging statements can easily be reconciled if it is realized that the Heisenberg inequalities refer to the initial state of the object, and, as already stressed a long time ago by Ballentine [7], do not refer to the measurement procedure (although, of course, the 
post-measurement state of the object will once again satisfy the Heisenberg inequalities if measurements are performed in this latter state). It should be realized, however, that in general there need not exist a direct relation between the determinative properties of a measurement, yielding information on the initial state of the object, and its preparative ones, determining what is the final object state.

The notion of a generalized measurement is able to clarify the confusion with respect to the role of the Heisenberg uncertainty relations in quantum measurement [8]. Whereas the standard formalism only allows the joint measurement of compatible (standard) observables, is the generalized formalism able to deal with incompatible ones. Moreover, from this formalism an inequality has been derived (equation (仿) below) quantifying the mutual disturbance of the information on the initial state, that is a consequence of the incompatibility of the observables measured jointly, and that, contrary to the Heisenberg inequality, is a property of the measurement procedure. In recent years quite a few measurement procedures have been analyzed satisfying the characteristics of such joint measurements: for instance, eight-port optical homodyning [9,10], certain neutron interference experiments [11], Stern-Gerlach experiments [12], and a number of Mach-Zehnder interferometric procedures [13] are of the generalized type, allowing an interpretation as a joint measurement of incompatible observables. Presumably also the experiment by Dürr et al. [6] is of this type, the observables measured jointly being different from the position-momentum pair.

In this paper we want to consider a recent atomic beam experiment [1] from the same point of view, and demonstrate that also this experiment has a generalized character. In the experiment, to be referred to in the following as the Haroche-Ramsey experiment, a $\mathrm{Rb}$ atom is sent through three cavities, $R_{1}, C$ and $R_{2}$ (figure 1 1 ), $R_{1}$ and $R_{2}$ being approximately resonant with a particular transition between two Rydberg states of the atom. Whereas the experiment without cavity $C$ is a pure interference experiment, already performed by Ramsey [14], the introduction of cavity $C$ provides the possibility to obtain also 'which way' information. The visibility of the interference fringes decreases as the field amplitude $\gamma$ increases, but it only vanishes in the limit $\gamma \rightarrow \infty$. So, for finite $\gamma$ information on both interference and path can be obtained. In order to actually obtain 'which way' information, a measurement must be carried out on the field in cavity $C$ left behind by the atom. The Haroche-Ramsey experiment is very analogous to the neutron interference experiments performed by Summhammer et al. 11, in which an absorber is inserted into one of the paths, the absorber playing an analogous role as the cavity $C$ field. Consequently the present analysis is similar to the analysis of the neutron interference experiments performed in [15].

Recently there has been much interest in the problem of reconstructing the initial state of the object on the basis of information provided by quantum measurements 16 - 19 . Such a reconstruction is impossible on the basis of a measurement of a single standard observable. As demonstrated by Vogel and Risken [20] in quantum tomography this goal may be achieved by measuring a number of such observables. Generalized measurements provide the opportunity to obtain a comparable result using one single measurement arrangement. In general a generalized measurement need not allow a complete reconstruction of the initial state. It then is an interesting question which information is provided by the measurement [21]. Also this subject will be discussed using the Haroche-Ramsey experiment as an example. The analysis is particularly suited to study the question of decoherence of the $\mathrm{C}$ field raised by Davidovich et al. [22]. In order to experimentally study the decoherence a second atom is sent through the cavities, yielding information on the state of the cavity $C$ field as it was at the time of passage. From the experimental two-atom correlation found in the experiment it was concluded [i] that a decoherence effect exists that cannot be explained by loss of photons from cavity $C$. From an analysis of the experiment as a generalized measurement it can be seen, however, that such a conclusion cannot be drawn, because as a measurement of the cavity $C$ field the measurement on the second atom can only provide non-ideal information on photon number.

The paper is organized as follows. In section II the theory of generalized measurements, and its application to joint non-ideal measurement of incompatible observables is briefly reviewed. In section III the Haroche-Ramsey experiment and the Davidovich-Haroche experiments are analyzed as generalized measurements. In section IV we demonstrate that the Davidovich-Haroche experiment is informationally equivalent to a Haroche-Ramsey experiment in which a measurement of cavity $C$ photon number is performed in coincidence with a determination of the final state of the atom. In this section the decoherence aspects of the Haroche-Ramsey experiment will be dealt with from the point of view of generalized measurements. In section $\mathrm{V}$ an alternative measurement procedure for the Haroche-Ramsey experiment is discussed, that can be interpreted as a joint measurement of incompatible observables having the complementary character of the "classical" double-slit experiments. 


\section{GENERALIZED MEASUREMENTS}

\section{A. Operational approach}

In the operational approach the experimental probabilities are calculated by treating the interaction between object and measuring instrument as a quantum mechanical process. Quantum mechanical measurement results are associated with pointer positions of the latter. If $\hat{\rho}$ and $\hat{\rho}_{a}$ are the initial density operators of object and measuring instrument, respectively, then the probability of a measurement result is obtained as the expectation value of the spectral representation $\left\{\hat{E}_{m}^{(a)}\right\}$ of a pointer observable of the measuring instrument in the final state $\hat{\rho}_{f}=\hat{U} \hat{\rho} \hat{\rho}_{a} \hat{U}^{\dagger}, \hat{U}=\exp \left(-i \hat{H} T_{f} / \hbar\right)$ of the measurement. Thus, $p_{m}=\operatorname{Tr}_{o a} \hat{\rho}_{f} \hat{E}_{m}^{(a)}$. This quantity can be interpreted as a property of the initial object state, $p_{m}=\operatorname{Tr}_{o} \hat{\rho} \hat{M}_{m}$, with $\hat{M}_{m}=\operatorname{Tr}_{a} \hat{\rho}_{a} \hat{U}^{\dagger} \hat{E}_{m}^{(a)} \hat{U}$. Whereas in the standard formalism quantum mechanical probabilities $p_{m}$ are represented by the expectation values of mutually commuting projection operators $\left(p_{m}=\operatorname{Tr} \hat{\rho} \hat{E}_{m}, \hat{E}_{m}^{2}=\hat{E}_{m},\left[\hat{E}_{m}, \hat{E}_{m^{\prime}}\right]_{-}=\hat{O}\right)$, allows the generalized formalism to represent these probabilities by expectation values of operators $\hat{M}_{m}$ that are not necessarily projection operators, and need not commute $\left(\hat{M}_{m}^{2} \neq \hat{M}_{m},\left[\hat{M}_{m}, \hat{M}_{m^{\prime}}\right]_{-} \neq \hat{O}\right.$ in general $)$. The operators $\hat{M}_{m}, \hat{O} \leq \hat{M}_{m} \leq \hat{I}, \sum_{m} \hat{M}_{m}=\hat{I}$ generate a POVM; the observables of the standard formalism are restricted to those POVMs of which the elements are mutually commuting projection operators (so-called projection-valued measures (PVM)).

\section{B. Non-ideal measurements}

In the generalized formalism it is possible to define a relation of partial ordering between observables, expressing that the measurement represented by one POVM can be interpreted as a non-ideal measurement of another one [8]. Thus, a POVM $\left\{\hat{R}_{m}\right\}$ is representing a non-ideal measurement of the (generalized or standard) observable $\left\{\hat{M}_{m^{\prime}}\right\}$ if the following relation holds between the elements of the POVMs:

$$
\hat{R}_{m}=\sum_{m^{\prime}} \lambda_{m m^{\prime}} \hat{M}_{m^{\prime}}, \lambda_{m m^{\prime}} \geq 0, \sum_{m} \lambda_{m m^{\prime}}=1 .
$$

The matrix $\left(\lambda_{m m^{\prime}}\right)$ is the non-ideality matrix. It is a so-called stochastic matrix. Its elements $\lambda_{m m^{\prime}}$ can be interpreted as conditional probabilities of finding measurement result $m$ if an ideal measurement had yielded measurement result $m^{\prime}$. In case of an ideal measurement the non-ideality matrix $\left(\lambda_{m m^{\prime}}\right)$ reduces to the unit matrix $\left(\delta_{m m^{\prime}}\right)$.

Non-ideality relations of type (1) are well-known from the theory of transmission channels in the classical theory of stochastic processes [23], where the non-ideality matrix describes the crossing of signals between subchannels. It should be noted, however, that, notwithstanding the classical origin of the latter subject, the non-ideality relation (1) may be of a quantum mechanical nature. Shannon's channel capacity can be used also in this latter case to quantify the deviation of a non-ideal measurement from ideality [8]. Another useful measure of the departure of a non-ideality matrix from the unit matrix is the average row entropy of the non-ideality matrix $\left(\lambda_{m m^{\prime}}\right)$,

$$
J_{(\lambda)}=-\frac{1}{N} \sum_{m m^{\prime}} \lambda_{m m^{\prime}} \ln \frac{\lambda_{m m^{\prime}}}{\sum_{m^{\prime \prime}} \lambda_{m m^{\prime \prime}}}
$$

which (restricting to square $N \times N$ matrices) satisfies the following properties:

$$
\begin{aligned}
& 0 \leq J_{(\lambda)} \leq \ln N \\
& J_{(\lambda)}=0 \text { if } \lambda_{m m^{\prime}}=\delta_{m m^{\prime}} \\
& J_{(\lambda)}=\ln N \text { if } \lambda_{m m^{\prime}}=\frac{1}{N} .
\end{aligned}
$$

Hence, the quantity $J_{(\lambda)}$ vanishes in case of an ideal measurement of observable $\left\{\hat{M}_{m^{\prime}}\right\}$, and obtains its maximal value if the measurement is uninformative (i.e. does not yield any information on the observable measured non-ideally; this is the case if in (11) $\lambda_{m m^{\prime}}$ is independent of $m^{\prime}$, and, hence, $\hat{R}_{m}=\alpha_{m} \hat{I}, \alpha_{m}$ constants for all $m$ ) due to maximal disturbance of the measurement results. In the following we shall use the non-ideality measure (2). 


\section{Joint non-deal measurement of incompatible observables}

Within the generalized formalism of POVMs it is possible to extend the notion of quantum mechanical measurement to the joint measurement of two (generalized) observables. Such a measurement is required to yield a bivariate joint probability distribution $p_{m n}$, satisfying $p_{m n} \geq 0, \sum_{m n} p_{m n}=1$. Here $m$ and $n$ label the possible values of the two observables measured jointly, corresponding to pointer positions of two different pointers (one for each observable) being jointly read for each individual preparation of an object. It is assumed that, analogous to the case of single measurement, the probabilities $p_{m n}$ of finding the pair $(m, n)$ are represented in the formalism by the expectation values $\operatorname{Tr} \hat{\rho} \hat{R}_{m n}$ of a bivariate POVM $\left\{\hat{R}_{m n}\right\}, \hat{R}_{m n} \geq \hat{O}, \sum_{m n} \hat{R}_{m n}=\hat{I}$ in the initial state $\hat{\rho}$ of the object. Then the marginal probabilities $\left\{\sum_{n} p_{m n}\right\}$ and $\left\{\sum_{m} p_{m n}\right\}$ are expectation values of POVMs $\left\{\hat{M}_{m}=\sum_{n} \hat{R}_{m n}\right\}$ and $\left\{\hat{N}_{n}=\sum_{m} \hat{R}_{m n}\right\}$, respectively, which correspond to the (generalized) observables measured jointly. A measurement, represented by a bivariate POVM $\left\{\hat{R}_{m n}\right\}$, can be interpreted as a joint non-ideal measurement of the observables $\left\{\hat{E}_{m}\right\}$ and $\left\{\hat{F}_{n}\right\}$ if the marginals $\left\{\sum_{n} \hat{R}_{m n}\right\}$ and $\left\{\sum_{m} \hat{R}_{m n}\right\}$ of the bivariate POVM $\left\{\hat{R}_{m n}\right\}$ represent non-ideal measurements of observables $\left\{\hat{E}_{m}\right\}$ and $\left\{\hat{F}_{n}\right\}$, respectively. Then, in accordance with (1) two non-ideality matrices $\left(\lambda_{m m^{\prime}}\right)$ and $\left(\mu_{n n^{\prime}}\right)$ exist, such that

$$
\begin{aligned}
& \sum_{n} \hat{R}_{m n}=\sum_{m^{\prime}} \lambda_{m m^{\prime}} \hat{E}_{m^{\prime}}, \lambda_{m m^{\prime}} \geq 0, \sum_{m} \lambda_{m m^{\prime}}=1, \\
& \sum_{m} \hat{R}_{m n}=\sum_{n^{\prime}} \mu_{n n^{\prime}} \hat{F}_{n^{\prime}}, \mu_{n n^{\prime}} \geq 0, \sum_{n} \mu_{n n^{\prime}}=1 .
\end{aligned}
$$

It is possible that $\left\{\hat{E}_{m}\right\}$ and $\left\{\hat{F}_{n}\right\}$ are standard observables. It will be demonstrated in the following sections that the Haroche-Ramsey experiment satisfies the joint measurement scheme given above.

If $\left\{\hat{E}_{m}\right\}$ and $\left\{\hat{F}_{n}\right\}$ are standard observables, then the non-idealities expressed by the non-ideality matrices $\left(\lambda_{m m^{\prime}}\right)$ and $\left(\mu_{n n^{\prime}}\right)$ can be proven [8] to satisfy the characteristic traits of the type of complementarity that is due to mutual disturbance of measurement results (or inaccuracy) in a joint measurement of incompatible observables. Quantifying the non-idealities of the non-ideality matrices $\left(\lambda_{m m^{\prime}}\right)$ and $\left(\mu_{n n^{\prime}}\right)$ by the average row entropy (2), it can be demonstrated [8] that for a joint non-ideal measurement of two standard observables $\hat{A}=\sum_{m} a_{m} \hat{E}_{m}$ and $\hat{B}=\sum_{n} b_{n} \hat{F}_{n}$, with eigenvectors $\left|a_{m}\right\rangle$ and $\left|b_{n}\right\rangle$, respectively, the non-ideality measures $J_{(\lambda)}$ and $J_{(\mu)}$ obey the following inequality:

$$
J_{(\lambda)}+J_{(\mu)} \geq-2 \ln \left\{\max _{m n}\left|\left\langle a_{m} \mid b_{n}\right\rangle\right|\right\} .
$$

It is evident that (19) is a nontrivial inequality (the right-hand side unequal to zero) if the two observables $\hat{A}$ and $\hat{B}$ are incompatible in the sense that the operators do not commute. Contrary to the Heisenberg inequality $\Delta \hat{A} \Delta \hat{B} \geq \frac{1}{2} \mid$ $\operatorname{Tr} \rho[\hat{A}, \hat{B}]_{-} \mid$, inequality (4) does not refer to the preparation of the initial state but exclusively to the measurement process. Inequality (11) should also be clearly distinguished from the entropic uncertainty relation 24 27] for the standard observables $\hat{A}=\sum_{m} a_{m} \hat{E}_{m}$ and $\hat{B}=\sum_{n} b_{n} \hat{F}_{n}$,

$$
H_{\left\{\hat{E}_{m}\right\}}(\hat{\rho})+H_{\left\{\hat{F}_{n}\right\}}(\hat{\rho}) \geq-2 \ln \left\{\max _{m n}\left|\left\langle a_{m} \mid b_{n}\right\rangle\right|\right\},
$$

in which $H_{\left\{\hat{E}_{m\}}\right.}(\hat{\rho})=-\sum_{m} p_{m} \ln p_{m}, p_{m}=\operatorname{Tr} \hat{\rho} \hat{E}_{m}$ (and analogously for $\hat{B}$ ). Inequality (5), although quite similar to inequality (1. tested by means of separate measurements of observables $\left\{\hat{E}_{m}\right\}$ and $\left\{\hat{F}_{n}\right\}$.

\section{Informational aspects}

The operators $\hat{M}_{m}$ of a POVM span a subspace $\mathcal{H}_{\left\{\hat{M}_{m}\right\}}$ of the linear space of bounded operators. For finitedimensional systems this can be the Hilbert-Schmidt space $\mathcal{H}_{H S}$ having inner product $\operatorname{Tr} \hat{A}^{\dagger} \hat{B}$. If the operators $\hat{M}_{m}$ are linearly independent they constitute a (generally non-orthogonal) basis of the subspace. Within subspace $\mathcal{H}_{\left\{\hat{M}_{m}\right\}}$ a bi-orthogonal system is defined by [21]

$$
\hat{M}_{m^{\prime}}^{\prime}=\sum_{m} \beta_{m^{\prime} m} \hat{M}_{m}, \operatorname{Tr} \hat{M}_{m} \hat{M}_{m^{\prime}}^{\prime}=\delta_{m m^{\prime}}, \beta_{m^{\prime} m} \text { real. }
$$

In general the set of Hermitian operators $\hat{M}_{m^{\prime}}^{\prime}$ constitutes another non-orthogonal basis of $\mathcal{H}_{\left\{\hat{M}_{m}\right\}}$. The information the measurement of POVM $\left\{\hat{M}_{m}\right\}$ yields on the initial state $\hat{\rho}$ of the object can be represented by the projection $\hat{\rho}_{\left\{\hat{M}_{m}\right\}}=\mathcal{P}_{\left\{\hat{M}_{m}\right\}} \hat{\rho}$ of $\hat{\rho}$ onto $\mathcal{H}_{\left\{\hat{M}_{m}\right\}}$. This projection is given by 


$$
\mathcal{P}_{\left\{\hat{M}_{m}\right\}} \hat{\rho}=\sum_{m}\left(\operatorname{Tr} \hat{M}_{m}^{\prime} \hat{\rho}\right) \hat{M}_{m}=\sum_{m}\left(\operatorname{Tr} \hat{M}_{m} \hat{\rho}\right) \hat{M}_{m}^{\prime}
$$

For complete measurements we have $\mathcal{P}_{\left\{\hat{M}_{m}\right\}} \hat{\rho}=\hat{\rho}$. Measurements are less complete as the subspace $\mathcal{H}_{\left\{\hat{M}_{m}\right\}}$ has a smaller dimension. It should be noted that, contrary to an assertion made in [21], for incomplete measurements $\hat{\rho}_{\left\{\hat{M}_{m}\right\}}$ need not be a density operator, even though $\operatorname{Tr} \hat{\rho}_{\left\{\hat{M}_{m}\right\}}=1$. In general $\hat{\rho}_{\left\{\hat{M}_{m}\right\}}$ is not a non-negative operator if the object Hilbert space has dimension greater than 2 (in the Appendix it is proven that for two-dimensional Hilbert spaces $\hat{\rho}_{\left\{\hat{M}_{m}\right\}}$ is non-negative). Hence, $\hat{\rho}_{\left\{\hat{M}_{m}\right\}}$ should be compared to a description of a quantum state by means of the Wigner distribution, yielding $\operatorname{Tr} \hat{\rho} \hat{A}=\operatorname{Tr} \hat{\rho}_{\left\{\hat{M}_{m}\right\}} \hat{A}$ for all operators $\hat{A} \in \mathcal{H}_{\left\{\hat{M}_{m}\right\}}$, but not containing any information on the part of $\hat{\rho}$ that is in the orthogonal complement of $\mathcal{H}_{\left\{\hat{M}_{m}\right\}}$. In principle the subspace $\mathcal{H}_{\left\{\hat{M}_{m}\right\}}$ completely determines the information on the initial density operator $\hat{\rho}$ that can be retrieved by a measurement of POVM $\left\{\hat{M}_{m}\right\}$.

\section{ATOMIC BEAM INTERFERENCE EXPERIMENTS}

\section{A. The Ramsey experiment}

In the Ramsey experiment [1] a beam of $\mathrm{Rb}$ atoms is sent through two identical cavities $R_{1}$ and $R_{2}$. The relevant Hilbert space $\mathcal{H}$ of a $\mathrm{Rb}$ atom is spanned by the orthogonal state vectors $|e\rangle$ and $|g\rangle$. These correspond to circular Rydberg levels with principal quantum numbers $n=51$ and $n=50$, respectively (transition frequency $\omega_{0}=\omega_{e}-\omega_{g}=$ $321 * 10^{9} \mathrm{rad} / \mathrm{s}$ ). The frequency of the classical microwave fields in the cavities is denoted by $\omega$, its amplitude by $\Omega$ (the Rabi frequency), and the time needed by an atom to pass one cavity by $T$. The unitary transformation $\hat{U}_{i}$ describing the evolution of the state of a $\mathrm{Rb}$ atom while passing cavity $R_{i}$ between $t=t_{i}$ and $t_{i}+T$ is given in the $\{|e\rangle,|g\rangle\}$-representation by the matrix

$$
\hat{U}_{i}=\left(\begin{array}{ll}
e^{-i\left(\frac{\nu}{2}+\omega_{e}\right) T} S_{1} & e^{-i\left(\frac{\nu}{2}+\omega_{e}\right) T-i \omega t_{i}} S_{2} \\
e^{i\left(\frac{\nu}{2}-\omega_{g}\right) T+i \omega t_{i}} S_{2} & e^{i\left(\frac{\nu}{2}-\omega_{g}\right) T} S_{1}^{*}
\end{array}\right)
$$

where $S_{1}=\cos \frac{a T}{2}+\frac{i \nu}{a} \sin \frac{a T}{2}, S_{2}=\frac{-i \Omega}{a} \sin \frac{a T}{2}, a=\sqrt{\nu^{2}+\Omega^{2}}$, and $\nu=\omega-\omega_{0}$ the detuning parameter. A derivation of (8) can be found in Ramsey [14] and Paul [28]. For all values of the parameters we have $\left|S_{1}\right|^{2}+\left|S_{2}\right|^{2}=1$. The Rb atom is said to undergo a $\frac{\pi}{2}$ pulse in cavity $R_{i}$ if $\left|S_{1}\right|^{2}=\left|S_{2}\right|^{2}=\frac{1}{2}$. We shall introduce a parameter $\delta=\left|S_{1}\right|^{2}-\left|S_{2}\right|^{2}$, quantifying experimental deviation from the $\frac{\pi}{2}$ pulse condition. Note that satisfaction of this latter condition does not imply $\nu=0$. The phase factor $e^{-i \omega t_{i}}$ in (8) takes into account the phase of the microwave field at the moment the atom enters the cavity.

Let $\left|\psi_{i n}\right\rangle$ be the initial state of the atom. If the standard observable $\{|e\rangle\langle e|| g\rangle,\langle g|\}$ is measured after the atom has passed cavity $R_{1}$, then the probabilities $p_{e}$ and $p_{g}$ can be related to the initial state by means of the equalities

$$
\begin{aligned}
& p_{e}=\left\langle\psi_{i n}\left|\left(\hat{U}_{1}^{\dagger}|e\rangle\langle e| \hat{U}_{1}\right)\right| \psi_{i n}\right\rangle=\left\langle\psi_{i n}\left|\hat{P}_{+}\right| \psi_{i n}\right\rangle, \\
& p_{g}=\left\langle\psi_{i n}\left|\left(\hat{U}_{1}^{\dagger}|g\rangle\langle g| \hat{U}_{1}\right)\right| \psi_{i n}\right\rangle=\left\langle\psi_{i n}\left|\hat{P}_{-}\right| \psi_{i n}\right\rangle .
\end{aligned}
$$

Due to the unitarity of $\hat{U}_{1}$ this yields PVM $\left\{\hat{P}_{+}, \hat{P}_{-}\right\}$as the POVM of this experiment, with

$$
\hat{P}_{+}=\left|p_{+}\right\rangle\left\langle p_{+}\left|=\hat{I}-\hat{P}_{-} ;\right| p_{+}\right\rangle=\left(\begin{array}{c}
S_{1}^{*} \\
S_{2}^{*} e^{i \omega t_{1}}
\end{array}\right),
$$

in which $\left|p_{+}\right\rangle$equals $\hat{U}_{1}^{\dagger}|e\rangle$ up to a phase factor. Because of the analogy with neutron interference experiments 15] the observable $\left\{\hat{P}_{+}, \hat{P}_{-}\right\}$will be referred to as the path observable, even though here the paths are not trajectories in configuration space (as it is in the double-slit experiment and the neutron interference experiments) but in the Hilbert space $\mathcal{H}$ of the internal states of the $\mathrm{Rb}$ atom. Mathematically this does not constitute a difference, however.

The observable $\left\{\hat{P}_{+}, \hat{P}_{-}\right\}$is dependent on the initial phase of the microwave field. For this reason it is not allowed to ignore this phase if the experiment is intended to yield a measurement of the initial state of the atom. If the atoms are prepared at random phases, then a measurement of $\{|e\rangle\langle e|| g\rangle,\langle g|\}$, performed immediately after the atom has 
passed cavity $R_{1}$, will yield probabilities obtained from (9) by phase averaging. The corresponding POVM $\left\{\overline{\hat{P}_{+}}, \overline{\hat{P}_{-}}\right\}$ is found according to

$$
\begin{aligned}
& \overline{\hat{P}_{+}}=\left|S_{1}\right|^{2}|e\rangle\left\langle\left. e|+| S_{2}\right|^{2} \mid g\right\rangle\langle g|, \\
& \overline{\hat{P}_{-}}=\left|S_{2}\right|^{2}|e\rangle\left\langle\left. e|+| S_{1}\right|^{2} \mid g\right\rangle\langle g|,
\end{aligned}
$$

and, hence, represents a non-ideal measurement of PVM $\{|e\rangle\langle e|| g\rangle,\langle g|\}$ in the sense of (11). Note that $\left\{\overline{\hat{P}_{+}}, \overline{\hat{P}_{-}}\right\}$is uninformative in case the $\frac{\pi}{2}$ pulse condition is satisfied, since then its expectation values do not yield any information on $\left|\psi_{\text {in }}\right\rangle$.

The Ramsey set-up consists of two cavities $R_{i}, i=1,2$, entered by the atom at times $t_{i}\left(\right.$ with $\left.t_{2}=t_{1}+T+\tau, \tau>0\right)$. If the initial state of the $\mathrm{Rb}$ atom is

$$
\left|\psi_{i n}\right\rangle=\alpha|e\rangle+\beta|g\rangle
$$

then the final state at time $t_{2}+T$ is:

$$
\left|\psi_{f}\right\rangle=[\alpha(E+F)+\beta(G+H)]|e\rangle+[\alpha(J+K)+\beta(L+M)]|g\rangle
$$

where we have used the abbreviations

$$
\begin{array}{ll}
E=S_{1}^{2} e^{-i\left(\nu+2 \omega_{e}\right) T-i \omega_{e} \tau} & J=S_{1} S_{2} e^{i \nu \tau} e^{i\left(\nu-2 \omega_{g}\right) T-i \omega_{g} \tau+i \omega t_{1}} \\
F=S_{2}^{2} e^{-i \nu \tau} e^{-i\left(\nu+2 \omega_{e}\right) T-i \omega_{e} \tau} & K=S_{1}^{*} S_{2} e^{i\left(\nu-2 \omega_{g}\right) T-i \omega_{g} \tau+i \omega t_{1}} \\
G=S_{1} S_{2} e^{-i\left(\nu+2 \omega_{e}\right) T-i \omega_{e} \tau-i \omega t_{1}} & L=S_{2}^{2} e^{i \nu \tau} e^{i\left(\nu-2 \omega_{g}\right) T-i \omega_{g} \tau} \\
H=S_{1}^{*} S_{2} e^{-i \nu \tau} e^{-i\left(\nu+2 \omega_{e}\right) T-i \omega_{e} \tau-i \omega t_{1}} & M=S_{1}^{* 2} e^{i\left(\nu-2 \omega_{g}\right) T-i \omega_{g} \tau}
\end{array}
$$

In the Ramsey experiment the standard observable $\{|e\rangle\langle e|| g\rangle,\langle g|\}$ is measured after the atom has passed cavity $R_{2}$. The corresponding probabilities $p_{e}$ and $p_{g}$ can be related to the initial state according to

$$
\begin{aligned}
& p_{e}=\left\langle\psi_{f} \mid e\right\rangle\left\langle e \mid \psi_{f}\right\rangle=\left\langle\psi_{i n}\left|\hat{Q}_{e}\right| \psi_{i n}\right\rangle, \\
& p_{g}=\left\langle\psi_{f} \mid g\right\rangle\left\langle g \mid \psi_{f}\right\rangle=\left\langle\psi_{i n}\left|\hat{Q}_{g}\right| \psi_{i n}\right\rangle,
\end{aligned}
$$

yielding

$$
\hat{Q}_{e}=\left|q_{e}\right\rangle\left\langle q_{e}\left|=\hat{I}-\hat{Q}_{g} ;\right| q_{e}\right\rangle=\left(\begin{array}{c}
\left(E^{*}+F^{*}\right) \\
\left(G^{*}+H^{*}\right)
\end{array}\right) .
$$

The observable $\left\{\hat{Q}_{e}, \hat{Q}_{g}\right\}$ can be interpreted as the quantum mechanical observable measured in the Ramsey experiment if the initial phase of the microwave field is well-defined. It is easily verified that $\left\langle q_{e} \mid q_{e}\right\rangle=1$. So $\hat{Q}_{e}$ and $\hat{Q}_{g}$ are projections and $\left\{\hat{Q}_{e}, \hat{Q}_{g}\right\}$ is a PVM. This PVM will be referred to as the interference observable, because, provided the atom velocity is sufficiently well-defined, its expectation values in the state $\left|\psi_{\text {in }}\right\rangle$ exhibit interference fringes if $\omega$ is varied. It is easily verified that if the $\frac{\pi}{2}$ pulse condition $\delta=0$ is satisfied, and the detuning parameter $\nu$ is taken to be zero, then the interference observable reduces to $\operatorname{PVM}\{|e\rangle\langle e|| g\rangle,\langle g|\}$. This is in agreement with the fact that under these conditions the Ramsey setup just interchanges the roles of the $e$ and $g$ states. In general the standard observables $\left\{\hat{P}_{+}, \hat{P}_{-}\right\}$and $\left\{\hat{Q}_{e}, \hat{Q}_{g}\right\}$ are incompatible.

Also $\left\{\hat{Q}_{e}, \hat{Q}_{g}\right\}$ is dependent on the initial phase of the microwave field. Averaging over this phase yields the POVM $\left\{\overline{\hat{Q}_{e}}, \overline{\hat{Q}_{g}}\right\}$,

$$
\begin{aligned}
& \overline{\hat{Q}_{e}}=\left|S_{1}^{2}+S_{2}^{2} e^{-i \nu \tau}\right|^{2}|e\rangle\left\langle\left. e|+| S_{2}\right|^{2}\left|S_{1}+S_{1}^{*} e^{-i \nu \tau}\right|^{2} \mid g\right\rangle\langle g|, \\
& \overline{\hat{Q}_{g}}=\hat{I}-\overline{\hat{Q}_{e}}
\end{aligned}
$$

which, once again, is a non-ideal measurement of $\{|e\rangle\langle e|| g\rangle,\langle g|\}$ (for $\nu=\delta=0$ it even is an ideal one). It is important to note that, nevertheless, the expectation values of $\left\{\hat{Q}_{e}, \overline{\hat{Q}_{g}}\right\}$ exhibit interference fringes if $\omega$ is varied. When in (11) $\beta=0$ (as was satisfied in the experiments that have actually been carried out), then the expectation values of $\hat{Q}_{e}$ and $\hat{Q}_{e}$ coincide. For this special case it is possible to analyze the Ramsey experiment in terms of the standard formalism, even if the actually performed experiment is a generalized one, described by POVM $\left\{\overline{\hat{Q}_{e}}, \overline{\hat{Q}_{g}}\right\}$. 
Contrary to the phase-averaged experiment, in case of a well-defined initial phase of the field the measurement performed after the atom left $R_{1}$ is incompatible with the one performed after $R_{2}$ if $\delta \neq \pm 1$. The two measurement arrangements are complementary. The experiments are analogous to double-slit experiments in which it either is directly measured which slit a particle has passed through ('which way' or path measurement), or the interference pattern is measured after the two partial beams have been allowed to interfere (interference experiment). The quantity $\nu \tau$ is the relative phase shift of the partial beams.

In standard quantum mechanics complementarity is interpreted as mutual exclusiveness of information, caused by the impossibility of having both experimental arrangements simultaneously. Of the two incompatible PVMs $\left\{\hat{P}_{+}, \hat{P}_{-}\right\}$ and $\left\{\hat{Q}_{e}, \hat{Q}_{g}\right\}$ either one or the other can be measured. In the following we shall discuss a measurement arrangement that is intermediate between the two arrangements considered above, viz. the experiment reported by Brune et al. [1], to be referred to as the Haroche-Ramsey experiment. As a result the experiment may yield information on both the path and the interference observable.

\section{B. The Haroche-Ramsey experiment}

In the Haroche-Ramsey experiment a third cavity $C$ is placed between cavities $R_{1}$ and $R_{2}$, storing a coherent field $|\gamma\rangle$. The transition frequency $\omega_{0}$ of the Rb atom and the frequency of the cavity $C$ field are chosen to be off-resonance, so there is no exchange of energy when the atom passes $C$. Contrary to the microwave fields in cavities $R_{1}$ and $R_{2}$ the cavity $C$ field is treated quantum mechanically. The field in cavity $C$ merely undergoes a phase shift $\Phi$ (single atom index effect) which depends on the state of the Rb atom in the following way [1]:

$$
|e\rangle \otimes|\gamma\rangle \stackrel{C}{\rightarrow}|e\rangle \otimes\left|\gamma e^{i \Phi}\right\rangle ;|g\rangle \otimes|\gamma\rangle \stackrel{C}{\rightarrow}|g\rangle \otimes\left|\gamma e^{-i \Phi}\right\rangle .
$$

The states $\left|\gamma e^{i \Phi}\right\rangle$ and $\left|\gamma e^{-i \Phi}\right\rangle$ are also coherent states. This yields the following unitary transformation $\hat{U}_{C}$ describing the evolution of the atom-field system when the atom is going from $R_{1}$ to $R_{2}$ :

$$
\hat{U}_{C}=e^{-i \omega_{e} \tau}|e\rangle\left\langle e\left|\otimes e^{i \Phi \hat{a}^{\dagger} \hat{a}}+e^{-i \omega_{g} \tau}\right| g\right\rangle\langle g| \otimes e^{-i \Phi \hat{a}^{\dagger} \hat{a}},
$$

where $\hat{a}^{\dagger}$ and $\hat{a}$ are the photon creation and annihilation operators of the cavity $C$ field mode. For the initial state $\left|\Psi_{i n}\right\rangle=[\alpha|e\rangle+\beta|g\rangle] \otimes|\gamma\rangle$ of the combined atom-field system we get as final state:

$$
\left|\Psi_{f}\right\rangle=\left\{\begin{array}{r}
|e\rangle \otimes\left[(\alpha E+\beta G)\left|\gamma e^{i \Phi}\right\rangle+(\alpha F+\beta H)\left|\gamma e^{-i \Phi}\right\rangle\right] \\
+|g\rangle \otimes\left[(\alpha J+\beta L)\left|\gamma e^{i \Phi}\right\rangle+(\alpha K+\beta M)\left|\gamma e^{-i \Phi}\right\rangle\right]
\end{array}\right.
$$

constants $E, G$, etc. being given by (12).

After the atom has passed cavity $C$ the field is containing path information that can be retrieved by a measurement of a well-chosen observable of the field. In the standard formalism this information is usually analyzed in terms of the inner product $\left\langle\gamma e^{i \Phi} \mid \gamma e^{-i \Phi}\right\rangle$ of the field states $\left|\gamma e^{i \Phi}\right\rangle$ and $\left|\gamma e^{-i \Phi}\right\rangle$, determining their distinguishability. The possibility of interference is seen as a consequence of the indistinguishability of the paths. How distinguishable the paths are, depends on the values of the parameters $\gamma$ and $\Phi$. If the states are identical, then the paths are completely indistinguishable. Ignoring the possibility $\Phi=m \pi(m \in \mathbb{Z})$, this obtains if $\gamma=0$. In this case the experiment cannot yield any path information. Complete distinguishability, corresponding to maximal path information, obtains if the field states are orthogonal. This only obtains in the limit $\gamma \rightarrow \infty$. In the next sections this analysis will be corroborated on the basis of the generalized formalism.

Whereas for the limiting values of $\gamma$, considered above, the standard formalism is sufficient, is the generalized formalism necessary for experiments corresponding to intermediate values $0<\gamma<\infty$. This already holds true if no measurement of the cavity $C$ field is carried out at all. Thus, putting $\left\langle\Psi_{f} \mid e\right\rangle\left\langle e \mid \Psi_{f}\right\rangle=\left\langle\psi_{i n}\left|\hat{R}_{e}\right| \psi_{i n}\right\rangle$ (and analogously for $g$ ) we find the POVM $\left\{\hat{R}_{e}, \hat{R}_{g}\right\}$ of the Haroche-Ramsey measurement from (16). Restricting to $\nu=\delta=0$ we get

$$
\hat{R}_{e}=\frac{1}{2}\left(\begin{array}{cc}
1-C_{1} & e^{-i \omega t_{1}} C_{2} \\
e^{i \omega t_{1}} C_{2} & 1+C_{1}
\end{array}\right), \hat{R}_{g}=\hat{I}-\hat{R}_{e}
$$

in which

$$
C_{1}+i C_{2}=\left\langle\gamma e^{i \Phi} \mid \gamma e^{-i \Phi}\right\rangle=e^{-\gamma^{2}\left(1-e^{-2 i \Phi}\right)}
$$


It is easily verified that, unless $C_{1}= \pm 1, C_{2}=0,\left\{\hat{R}_{e}, \hat{R}_{g}\right\}$ is not a PVM. Even in the limit $\gamma \rightarrow \infty$ (corresponding to $\left.C_{1}=C_{2}=0\right)(17)$ is a POVM, be it an uninformative one. This is consistent with complementarity in the sense that in this limit no information on the interference observable is obtained, path information being obtainable by a measurement of an observable of the cavity $C$ field in the final state of this field.

Since the operators of POVM $\left\{\overline{\hat{R}_{e}}, \hat{R}_{g}\right\}$, obtained from (17) by phase-averaging, are diagonal, the Haroche-Ramsey experiment is just a non-ideal version of the Ramsey experiment if the initial phase of the microwave field is random. The non-ideality measure (2) is then given as

$$
J^{\overline{H R}}=-\frac{\left(1-C_{1}\right)}{2} \ln \frac{\left(1-C_{1}\right)}{2}-\frac{\left(1+C_{1}\right)}{2} \ln \frac{\left(1+C_{1}\right)}{2} .
$$

This quantity is a measure of the inaccuracy introduced in the observation of observable $\{|e\rangle\langle e|| g\rangle,\langle g|\}$ by the insertion of cavity $C$.

From the point of view of complementarity the experimental setup of the Haroche-Ramsey experiment is particularly interesting when also the information is exploited that is stored in the cavity $C$ field, because this may add "which way' information to the (non-ideal) interference information obtained from the measurement of the final state of the atom. This will be discussed in the next sections.

\section{The Davidovich-Haroche experiment}

By Davidovich et al. 22] a variation of the Haroche-Ramsey experiment has been proposed in which a second atom traverses the system some time after the first one has passed. In 22] the reason for sending this second atom is to probe a possible decoherence of the field in cavity $C$. We shall discuss this aspect of the experiment in section IV A. Here we are interested in the possibility to consider the second atom as yielding information on the cavity $C$ field that might be useful for determining the path of atom 1 . We shall demonstrate that the joint measurement of standard observables $\left\{\left|e_{1}\right\rangle\left\langle e_{1}|,| g_{1}\right\rangle\left\langle g_{1}\right|\right\}$ and $\left\{\left|e_{2}\right\rangle\left\langle e_{2}|,| g_{2}\right\rangle\left\langle g_{2}\right|\right\}$ in the final state of the atoms can be interpreted as a measurement of a POVM on the incoming state of atom 1 . We shall neglect decoherence here by taking a negligible time interval between the atoms. We also restrict here to the case $\nu=\delta=0$, for which $E+F=0$, and, hence, (13) is yielding $\hat{Q}_{e}=|g\rangle\left\langle g\left|, \hat{Q}_{g}=\right| e\right\rangle\langle e|$. Then, starting with atom 2 in state $\left|e_{2}\right\rangle$, and using rules (14) for both atoms, we find for an arbitrary initial state $\left|\psi_{i n 1}\right\rangle=\alpha\left|e_{1}\right\rangle+\beta\left|g_{1}\right\rangle$ of atom 1 the final state

$$
\left|\Psi_{f}\right\rangle=\frac{1}{4}\left[\begin{array}{c}
\left|e_{1} e_{2}\right\rangle e^{-2 i \omega T}\left\{\alpha\left(\left|v_{g}^{\prime}\right\rangle-2|\gamma\rangle\right)-i \beta e^{-i \omega t_{1}}\left|v_{e}^{\prime}\right\rangle\right\}+ \\
\left|e_{1} g_{2}\right\rangle e^{i \omega_{0} \tau}\left\{-i \alpha e^{i \omega t_{1}}\left|v_{e}^{\prime}\right\rangle-\beta\left(\left|v_{g}^{\prime}\right\rangle+2|\gamma\rangle\right)\right\}+ \\
\left|g_{1} e_{2}\right\rangle e^{i \omega_{0} \tau}\left\{-i \alpha e^{i \omega t_{1}}\left|v_{e}^{\prime}\right\rangle-\beta\left(\left|v_{g}^{\prime}\right\rangle-2|\gamma\rangle\right)\right\}+ \\
\left|g_{1} g_{2}\right\rangle e^{2 i \omega T+2 i \omega_{0} \tau+i \omega t_{1}}\left\{-\alpha e^{i \omega t_{1}}\left(\left|v_{g}^{\prime}\right\rangle+2|\gamma\rangle\right)+i \beta\left|v_{e}^{\prime}\right\rangle\right\}
\end{array}\right],
$$

with $\left|v_{e}^{\prime}\right\rangle=\left|\gamma e^{i 2 \Phi}\right\rangle-\left|\gamma e^{-i 2 \Phi}\right\rangle,\left|v_{g}^{\prime}\right\rangle=\left|\gamma e^{i 2 \Phi}\right\rangle+\left|\gamma e^{-i 2 \Phi}\right\rangle$.

By putting $\left\langle\Psi_{f}|| e_{1}\right\rangle\left\langle e_{1}|\otimes| e_{2}\right\rangle\left\langle e_{2}|| \Psi_{f}\right\rangle=\left\langle\psi_{i n 1}\left|\hat{M}_{e_{1} e_{2}}\right| \psi_{i n 1}\right\rangle$, etc., the POVM of the Davidovich-Haroche experiment, interpreted as a measurement on atom 1 , is straightforwardly found according to

$$
\begin{aligned}
& \hat{M}_{e_{1} e_{2}}=\frac{1}{16}\left(\begin{array}{cc}
\|\left|v_{g}^{\prime}\right\rangle-2|\gamma\rangle \|^{2} & -i e^{-i \omega t_{1}}\left(\left\langle v_{g}^{\prime} \mid v_{e}^{\prime}\right\rangle-2\left\langle\gamma \mid v_{e}^{\prime}\right\rangle\right) \\
i e^{i \omega t_{1}}\left(\left\langle v_{e}^{\prime} \mid v_{g}^{\prime}\right\rangle-2\left\langle v_{e}^{\prime} \mid \gamma\right\rangle\right) & \|\left|v_{e}^{\prime}\right\rangle \|^{2}
\end{array}\right), \\
& \hat{M}_{e_{1} g_{2}}=\frac{1}{16}\left(\begin{array}{cc}
\|\left|v_{e}^{\prime}\right\rangle \|^{2} & -i e^{-i \omega t_{1}}\left(\left\langle v_{e}^{\prime} \mid v_{g}^{\prime}\right\rangle+2\left\langle v_{e}^{\prime} \mid \gamma\right\rangle\right) \\
i e^{i \omega t_{1}}\left(\left\langle v_{g}^{\prime} \mid v_{e}^{\prime}\right\rangle+2\left\langle\gamma \mid v_{e}^{\prime}\right\rangle\right) & \|\left|v_{g}^{\prime}\right\rangle+2|\gamma\rangle \|^{2}
\end{array}\right), \\
& \hat{M}_{g_{1} e_{2}}=\frac{1}{16}\left(\begin{array}{cc}
\|\left|v_{e}^{\prime}\right\rangle \|^{2} & -i e^{-i \omega t_{1}}\left(\left\langle v_{e}^{\prime} \mid v_{g}^{\prime}\right\rangle-2\left\langle v_{e}^{\prime} \mid \gamma\right\rangle\right) \\
i e^{i \omega t_{1}}\left(\left\langle v_{g}^{\prime} \mid v_{e}^{\prime}\right\rangle-2\left\langle\gamma \mid v_{e}^{\prime}\right\rangle\right) & \|\left|v_{g}^{\prime}\right\rangle-2|\gamma\rangle \|^{2}
\end{array}\right), \\
& \hat{M}_{g_{1} g_{2}}=\frac{1}{16}\left(\begin{array}{cc}
\|\left|v_{g}^{\prime}\right\rangle+2|\gamma\rangle \|^{2} & -i e^{-i \omega t_{1}}\left(\left\langle v_{g}^{\prime} \mid v_{e}^{\prime}\right\rangle+2\left\langle\gamma \mid v_{e}^{\prime}\right\rangle\right) \\
i e^{i \omega t_{1}}\left(\left\langle v_{e}^{\prime} \mid v_{g}^{\prime}\right\rangle+2\left\langle v_{e}^{\prime} \mid \gamma\right\rangle\right) & \|\left|v_{e}^{\prime}\right\rangle \|^{2}
\end{array}\right) .
\end{aligned}
$$

From these expressions it is immediately clear that averaging over the initial phase $\omega t_{1}$ makes also the DavidovichHaroche experiment a non-ideal measurement of observable $\{|e\rangle\langle e|| g\rangle,\langle g|\}$, the non-ideality measure (2) being given by

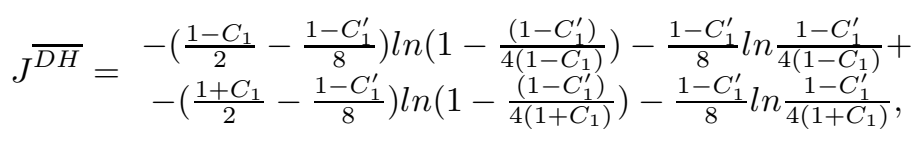


with

$$
C_{1}^{\prime}+i C_{2}^{\prime}=\left\langle\gamma e^{i 2 \Phi} \mid \gamma e^{-i 2 \Phi}\right\rangle .
$$

Comparing $J^{\overline{D H}}$ with the corresponding non-ideality measure (19) of the Haroche-Ramsey experiment, we find (cf. figure 21) that $J^{\overline{D H}}<J^{\overline{H R}}$ for $\gamma \neq 0$. Hence, by taking into account the extra information from the measurement of the cavity $C$ field the quality of the non-ideal measurement of the interference observable $\left\{\hat{Q}_{e}, \hat{Q}_{g}\right\}$ has been increased.

In the phase-averaged case the subspace $\mathcal{H}_{\left\{\hat{M}_{m}\right\}}$ of Hilbert-Schmidt space spanned by the operators of POVM (20) is a two-dimensional one. From an informational point of view the Davidovich-Haroche experiment will be more interesting if it is possible to avoid the necessity of phase averaging, because in that case $\mathcal{H}_{\left\{\hat{M}_{m}\right\}}$ is three-dimensional. Although, due to the equality $\hat{M}_{e_{1} e_{2}}+\hat{M}_{g_{1} e_{2}}=\left(\|\left|v_{g}^{\prime}\right\rangle-2|\gamma\rangle\left\|^{2}+\right\|\left|v_{e}^{\prime}\right\rangle \|^{2}\right) / 16 * \hat{I}$, the operators of the POVM are linearly dependent, and, hence, the measurement is not a complete one, it nevertheless is a generalized measurement, being interpretable, in the sense defined in sect. II G, as a joint non-ideal measurement of two incompatible observables. In order to see this the operators must be ordered in a bivariate way. Due to the uninformativeness of the marginal $\left\{\hat{M}_{e_{1} e_{2}}+\hat{M}_{g_{1} e_{2}}, \hat{M}_{e_{1} g_{2}}+\hat{M}_{g_{1} g_{2}}\right\}$ the only interesting way to do so is according to

$$
\hat{R}_{m n}=\left(\begin{array}{cc}
\hat{M}_{e_{1} e_{2}} & \hat{M}_{e_{1} g_{2}} \\
\hat{M}_{g_{1} g_{2}} & \hat{M}_{g_{1} e_{2}}
\end{array}\right)
$$

yielding marginals $\left\{\Sigma_{n} \hat{R}_{m n}\right\}$ and $\left\{\Sigma_{m} \hat{R}_{m n}\right\}$ with

$$
\hat{M}_{e_{1} e_{2}}+\hat{M}_{e_{1} g_{2}}=\frac{1}{2}\left(\begin{array}{cc}
1-C_{1} & C_{2} \\
C_{2} & 1+C_{1}
\end{array}\right) \text { and } \hat{M}_{e_{1} e_{2}}+\hat{M}_{g_{1} g_{2}}=\frac{1}{4}\left(\begin{array}{cc}
3+C_{1}^{\prime} & -C_{2}^{\prime} \\
-C_{2}^{\prime} & 1-C_{1}^{\prime}
\end{array}\right) \text {. Here } t_{1} \text { is taken to be zero. }
$$

Evidently, both marginals are depending on the parameter $\Phi$ governing the distinguishability of the field states. In agreement with (3), for $\gamma \neq 0$ these marginals can be interpreted as describing non-ideal measurements of two incompatible PVMs of atom 1, with non-ideality matrices given by

$$
\begin{aligned}
(\lambda) & =\left(\begin{array}{cc}
\lambda & 1-\lambda \\
1-\lambda & \lambda
\end{array}\right),(\mu)=\left(\begin{array}{cc}
\mu & 1-\mu \\
1-\mu & \mu
\end{array}\right), \\
\lambda & =\frac{1}{2}\left(1+\sqrt{C_{1}^{2}+C_{2}^{2}}\right), \mu=\frac{1}{2}\left(1+\frac{1}{2} \sqrt{\left(C_{1}^{\prime}+1\right)^{2}+C_{2}^{\prime 2}}\right),
\end{aligned}
$$

yielding non-ideality measures (21) as

$$
\begin{aligned}
& J_{(\lambda)}=-\{\lambda \ln (\lambda)+(1-\lambda) \ln (1-\lambda)\}, \\
& J_{(\mu)}=-\{\mu \ln (\mu)+(1-\mu) \ln (1-\mu)\} .
\end{aligned}
$$

For the parameters $\lambda$ and $\mu$ we find

$$
\begin{aligned}
& \lambda=\frac{1}{2}\left(1+e^{-2 \gamma^{2} \sin ^{2} \Phi}\right), \\
& \mu=\frac{1}{2}+\frac{1}{4}\left[\left\{1+e^{-2 \gamma^{2} \sin ^{2} 2 \Phi} \cos \left(\gamma^{2} \sin 4 \Phi\right)\right\}^{2}+e^{-4 \gamma^{2} \sin ^{2} 2 \Phi} \sin ^{2}\left(\gamma^{2} \sin 4 \Phi\right)\right]^{1 / 2} .
\end{aligned}
$$

We shall not bother to calculate the corresponding PVMs, because these do not admit a straightforward physical interpretation in terms of the interference and path observables defined above. From the non-ideality measures $J_{(\lambda)}$ and $J_{(\mu)}$ it can already be seen that the two PVMs measured jointly in the Davidovich-Haroche experiment do not constitute a canonically conjugate pair in the sense that, if the parameter $\gamma$ is varied, one measurement gets more accurate if the other one becomes more non-ideal. Thus, in both of the limits $\gamma=0$ and $\gamma \rightarrow \infty$ POVM (20) is representing a (non-)ideal measurement of the same PVM $\{|e\rangle\langle e|| g\rangle,\langle g|\}$. From the plots of $J_{(\lambda)}$ and $J_{(\mu)}$ as functions of $\gamma$ and $\Phi$ in figure 3 it is also seen that both non-ideality measures vanish in the limit $\gamma \rightarrow 0$. Hence, although the two PVMs measured jointly in this experiment do satisfy inequality (4) for all values of $\gamma$, this does not imply any complementarity for $\gamma \rightarrow 0$ because the right-hand side of the inequality is vanishing in this limit due to the fact that the two PVMs coincide. As will be demonstrated in section IV, the Davidovich-Haroche experiment, for general values of the parameters $\nu$ and $\delta$, is informationally equivalent to a measurement in which the second atom is replaced by a measurement of photon number in the final state of cavity $C$. The absence of information on the phase of the cavity $C$ field explains the somewhat non-complementary behavior observed here. In section $\square$ an alternative measurement procedure will be discussed, better satisfying the canonical notion of complementarity, in which it is proposed to perform a measurement of the cavity $C$ field also yielding phase information. 


\section{INFORMATIONAL ASPECTS OF THE DAVIDOVICH-HAROCHE EXPERIMENT}

\section{A. Decoherence}

The Haroche-Ramsey experiment [1] was devised in the first place to probe decoherence in cavity $C$ following the passage of a $\mathrm{Rb}$ atom, entering in state $|e\rangle$. Hence $\beta=0$ in the final state (16). Restricting to $\nu=\delta=0$ it follows from (16) that, conditional on measurement result $e$ or $g$, the cavity $C$ field is described by a superposition of coherent states $\left(e \rightarrow\left|v_{e}\right\rangle=\left|\gamma e^{i \Phi}\right\rangle-\left|\gamma e^{-i \Phi}\right\rangle, g \rightarrow\left|v_{g}\right\rangle=\left|\gamma e^{i \Phi}\right\rangle+\left|\gamma e^{-i \Phi}\right\rangle\right)$. These states can be considered as Schrödinger cat states if $\gamma$ is sufficiently large. In 22] it was proposed to probe, by sending after a time $T$ a second Rb atom through the system (like the first atom starting in state $|e\rangle$ ), whether a process of decoherence is active by which these superpositions could decay to a mixture of the coherent states $\left|\gamma e^{i \Phi}\right\rangle$ and $\left|\gamma e^{-i \Phi}\right\rangle$. In [1] it was concluded from the observed two-atom correlations that a decoherence effect obtains. In particular it was inferred from the experimental data that the decoherence time is much shorter than the decay time that can be attributed to loss of photons from cavity $C$. In the present section this latter conclusion is challenged. It is demonstrated that, in agreement with a result obtained by Vitali et al. 29], the second $\mathrm{Rb}$ atom can only yield information on cavity $C$ 's photon number. Hence, any change of the measurement results obtained for this atom should be attributed to a change of photon number. Of course, this does not imply the absence of decoherence due to decay of pure phase correlations. However, this measurement is not sensitive to it.

In order to demonstrate this we have to determine which information is obtained on the cavity $C$ field by the measurement of the second atom. The corresponding POVM can be found by equating, for an arbitrary initial coherent state $|\alpha\rangle$ of the cavity field, the final state probabilities $p_{e}$ and $p_{g}$ to expectation values $\left\langle\alpha\left|\hat{M}_{e}\right| \alpha\right\rangle$ and $\langle\alpha| \hat{M}_{g}$ $|\alpha\rangle$, respectively. With $\left|\Psi_{f}\right\rangle=\frac{1}{2}\left|e_{2}\right\rangle\left(E\left|\alpha e^{i \Phi}\right\rangle+F\left|\alpha e^{-i \Phi}\right\rangle\right)+\frac{1}{2}\left|g_{2}\right\rangle\left(J\left|\alpha e^{i \Phi}\right\rangle+K\left|\alpha e^{-i \Phi}\right\rangle\right)$ we find, once again restricting to $\nu=\delta=0$,

$$
\begin{aligned}
& p_{e}=\left\langle\Psi_{f} \mid e_{2}\right\rangle\left\langle e_{2} \mid \Psi_{f}\right\rangle=\frac{1}{4} \|\left|\alpha e^{i \Phi}\right\rangle-\left|\alpha e^{-i \Phi}\right\rangle \|^{2}=\frac{1}{4}\left\langle\alpha\left|2-e^{2 i \Phi \hat{a}^{\dagger} \hat{a}}-e^{-2 i \Phi \hat{a}^{\dagger} \hat{a}}\right| \alpha\right\rangle, \\
& p_{g}=\left\langle\Psi_{f} \mid g_{2}\right\rangle\left\langle g_{2} \mid \Psi_{f}\right\rangle=\frac{1}{4} \|\left|\alpha e^{i \Phi}\right\rangle+\left|\alpha e^{-i \Phi}\right\rangle \|^{2}=\frac{1}{4}\left\langle\alpha\left|2+e^{2 i \Phi \hat{a}^{\dagger} \hat{a}}+e^{-2 i \Phi \hat{a}^{\dagger} \hat{a}}\right| \alpha\right\rangle,
\end{aligned}
$$

from which we obtain

$$
\hat{M}_{e}=\sin ^{2} \Phi \hat{a}^{\dagger} \hat{a}, \hat{M}_{g}=\cos ^{2} \Phi \hat{a}^{\dagger} \hat{a}
$$

Note that this result holds independent of phase averaging. It is easily seen that POVM $\left\{\hat{M}_{e}, \hat{M}_{g}\right\}$ represents a non-ideal measurement of the number observable $\hat{N}=\hat{a}^{\dagger} \hat{a}=\sum_{n=0}^{\infty} n|n\rangle\langle n|$ in the sense of definition (1):

$$
\begin{aligned}
\hat{M}_{e} & =\sum_{n=0}^{\infty} \lambda_{e n}|n\rangle\left\langle n\left|, \hat{M}_{g}=\sum_{n=0}^{\infty} \lambda_{g n}\right| n\right\rangle\langle n|, \\
\lambda_{e n} & =\sin ^{2} \Phi n, \lambda_{g n}=\cos ^{2} \Phi n .
\end{aligned}
$$

Using (6) it is possible to calculate the projection $\hat{\rho}_{\left\{\hat{M}_{m}\right\}}$ of the density operator $\hat{\rho}$ on the subspace spanned by $\hat{M}_{e}$ and $\hat{M}_{g}$, representing the information that is obtained by a measurement of POVM $\left\{\hat{M}_{e}, \hat{M}_{g}\right\}$. Due to the infinitedimensionality of the Hilbert space of the field this must be done with some care because the operators are not Hilbert-Schmidt operators then. For this reason the dimension must be truncated. Restricting to an arbitrary large but finite value $D$, we get:

$$
\begin{aligned}
\hat{M}_{m}^{\prime} & =\sum_{n=0}^{D}\left[\beta_{m e} \sin ^{2} \Phi n+\beta_{m g} \cos ^{2} \Phi n\right]|n\rangle\langle n|, m=e, g, \\
\beta_{e e} & =\frac{r-s}{(1+r-s)(D-r-s)-s^{2}}, \beta_{g e}=\beta_{e g}=\frac{-s}{(1+r-s)(D-r-s)-s^{2}}, \\
\beta_{g g} & =\frac{D-r-s}{(1+r-s)(D-r-s)-s^{2}},
\end{aligned}
$$

with $r=\sum_{n=1}^{D} \cos ^{2} \Phi n, s=\sum_{n=1}^{D} \sin ^{2} \Phi n \cos ^{2} \Phi n$. Then 


$$
\begin{aligned}
\hat{\rho}_{\left\{\hat{M}_{m}\right\}}= & \lim _{D \rightarrow \infty} \sum_{n, n^{\prime}=0}^{D}\left[\left(\beta_{e e} \sin ^{2} \Phi n^{\prime}+\beta_{g e} \cos ^{2} \Phi n^{\prime}\right) \sin ^{2} \Phi n+\right. \\
& \left.+\left(\beta_{e g} \sin ^{2} \Phi n^{\prime}+\beta_{g g} \cos ^{2} \Phi n^{\prime}\right) \cos ^{2} \Phi n\right]\left\langle n^{\prime}|\hat{\rho}| n^{\prime}\right\rangle|n\rangle\langle n| .
\end{aligned}
$$

Note that, although $\beta_{m m^{\prime}} \rightarrow 0$ if $D \rightarrow \infty$, yet $\hat{\rho}_{\left\{\hat{M}_{m\}}\right.} \neq \hat{O}$. Thus, it is easily verified that also in the limit $D \rightarrow \infty$ $\operatorname{Tr} \hat{\rho}_{\left\{\hat{M}_{m}\right\}}=1$, and $\operatorname{Tr} \hat{\rho}_{\left\{\hat{M}_{m}\right\}} \hat{M}_{m}=\operatorname{Tr} \hat{\rho} \hat{M}_{m}, m=e, g$, the latter equality explicitly demonstrating that $\hat{\rho}_{\left\{\hat{M}_{m}\right\}}$ contains the same information on the measurement results of POVM $\left\{\hat{M}_{e}, \hat{M}_{g}\right\}$ as does $\hat{\rho}$.

Although a measurement of this POVM can distinguish between a mixture of the states $\left|v_{e}\right\rangle$ and $\left|v_{g}\right\rangle$ and a mixture of the states $\left|\gamma e^{i \Phi}\right\rangle$ and $\left|\gamma e^{-i \Phi}\right\rangle$, this is only so because the probability distributions of the photon number observable are different in the two mixtures. Decoherence, not accompanied by a change of photon number, cannot be observed using the Davidovich-Haroche experiment.

\section{B. Informational equivalence of second atom and number measurement}

In this section it will be demonstrated that the Davidovich-Haroche experiment, in which a second atom is used as a probe of the cavity $C$ field, is informationally equivalent to a Haroche-Ramsey experiment in which the 'which-way' information is obtained by measuring photon number. This will be done by considering the informational aspects of the measurement, introduced in section IID. From the informational point of view the important feature is the structure of the subspaces $\mathcal{H}_{\left\{\hat{M}_{m}\right\}}$ of Hilbert-Schmidt space, spanned by the operators $\hat{M}_{m}$ generating the POVM, as a function of the experimental parameters. In order to be completely general, in this section we allow the different parameters to take arbitrary values. We first determine the POVM of the Haroche-Ramsey experiment in which cavity $C$ photon number is measured in coincidence with a determination of the final state of the atom. This POVM is found from (16) by the equalities

$$
\begin{aligned}
& p_{e n}=\left\langle\Psi_{f} \mid e\right\rangle\langle e|\otimes| n\rangle\left\langle n \mid \Psi_{f}\right\rangle=\left\langle\psi_{i n}\left|\hat{M}_{e n}\right| \psi_{i n}\right\rangle, \\
& p_{g n}=\left\langle\Psi_{f} \mid g\right\rangle\langle g|\otimes| n\rangle\left\langle n \mid \Psi_{f}\right\rangle=\left\langle\psi_{i n}\left|\hat{M}_{g n}\right| \psi_{i n}\right\rangle,
\end{aligned}
$$

in which $p_{e n}$ and $p_{g n}$ are the measured joint probabilities, and $\{|n\rangle\langle n|\}$ is the PVM corresponding to the spectral measure of the photon number observable. With $t_{1}=0$ the operators $\hat{M}_{e n}$ and $\hat{M}_{g n}$ are found as

$$
\begin{aligned}
& \hat{M}_{e n}=\frac{e^{-\gamma^{2}} \gamma^{2 n}}{n !}\left(\begin{array}{cc}
\sin ^{2} \frac{\varphi_{n}}{2}+\delta^{2} \cos ^{2} \frac{\varphi_{n}}{2} & -e^{-i \psi} \frac{\sqrt{1-\delta^{2}}}{2}\left[\sin \varphi_{n}+2 i \delta \cos ^{2} \frac{\varphi_{n}}{2}\right] \\
-e^{i \psi} \frac{\sqrt{1-\delta^{2}}}{2}\left[\sin \varphi_{n}-2 i \delta \cos ^{2} \frac{\varphi_{n}}{2}\right] & \left(1-\delta^{2}\right) \cos ^{2} \frac{\varphi_{n}}{2}
\end{array}\right), \\
& \hat{M}_{g n}=\frac{e^{-\gamma^{2}} \gamma^{2 n}}{n !}\left(\begin{array}{cc}
\left(1-\delta^{2}\right) \cos ^{2} \frac{\varphi_{n}}{2} & e^{-i \psi} \frac{\sqrt{1-\delta^{2}}}{2}\left[\sin \varphi_{n}+2 i \delta \cos ^{2} \frac{\varphi_{n}}{2}\right] \\
e^{i \psi \frac{\sqrt{1-\delta^{2}}}{2}}\left[\sin \varphi_{n}-2 i \delta \cos ^{2} \frac{\varphi_{n}}{2}\right] & \sin ^{2} \frac{\varphi_{n}}{2}+\delta^{2} \cos ^{2} \frac{\varphi_{n}}{2}
\end{array}\right),
\end{aligned}
$$

in which $\varphi_{n}=2 n \Phi+\nu \tau+2 \psi, \psi=\arg \left(S_{1}\right)$.

Excluding $\delta= \pm 1$, for which POVM $\left\{\hat{M}_{e n}, \hat{M}_{g n}\right\}$ reduces to a trivial refinement of PVM $\{|e\rangle\langle e|| g\rangle,\langle g|\}$, for most values of the parameters the operators $\hat{M}_{e n}$ and $\hat{M}_{g n}, n=0,1$. span the whole Hilbert-Schmidt space of operators on a 2-dimensional Hilbert space. Hence, in general the measurement is a complete measurement. The parameter values for which the measurement is incomplete can be found by looking for Hermitian operators $\hat{T}$ that are orthogonal to all $\hat{M}_{e n}$ and $\hat{M}_{g n}$. Thus, $\operatorname{Tr} \hat{M}_{e n} \hat{T}=\operatorname{Tr} \hat{M}_{g n} \hat{T}=0, n=0, .$. We find

$\Phi=\frac{\pi}{2}, \delta \neq 0$ :

$\hat{T}=\left(\begin{array}{cc}-\sqrt{1-\delta^{2}} \tan (\nu \tau+2 \psi) & e^{-i \psi}[1-i \delta \tan (\nu \tau+2 \psi)] \\ e^{i \psi}[1+i \delta \tan (\nu \tau+2 \psi)] & \sqrt{1-\delta^{2}} \tan (\nu \tau+2 \psi)\end{array}\right)$

$\Phi \neq \frac{\pi}{2}, \delta=0$ :

$\hat{T}=\left(\begin{array}{cc}0 & i e^{-i \psi} \\ -i e^{i \psi} & 0\end{array}\right)$;

$\Phi=\frac{\pi}{2}, \delta=0$ :

$\hat{T}=\left(\begin{array}{cc}\tan (\nu \tau+2 \psi) & e^{-i \psi} \\ e^{i \psi} & \tan (\nu \tau+2 \psi)\end{array}\right),\left(\begin{array}{cc}0 & i e^{-i \psi} \\ -i e^{i \psi} & 0\end{array}\right)$. Barring $\delta= \pm 1$, for all other values of the parameters no solution for $\hat{T}$ can be found. Hence, specializing the parameters of the experiment to either $\Phi=\frac{\pi}{2}$, or to the 
$\frac{\pi}{2}$ pulse condition $\delta=0$, reduces the dimension of the subspace spanned by the operators of the POVM to 3 , the dimensionality being further reduced if both conditions are simultaneously satisfied. By determining in the same way the Hilbert-Schmidt operators that are orthogonal to the operators of POVM (20) it is straightforward to prove that the Davidovich-Haroche experiment, discussed in section III C, has exactly the same structure of subspaces, thus demonstrating the informational equivalence of these experiments for all values of the parameters.

The subspace structure is not essentially changed by taking the detuning parameter $\nu=0$. Since then also $\psi=0$ the operators $\hat{T}$, found above, are particularly simple, viz. $\left(\begin{array}{cc}0 & i \\ -i & 0\end{array}\right)$ and $\left(\begin{array}{ll}0 & 1 \\ 1 & 0\end{array}\right)$. These are two orthogonal vectors, constituting together with the operators $\left(\begin{array}{ll}1 & 0 \\ 0 & 0\end{array}\right)$ and $\left(\begin{array}{ll}0 & 0 \\ 0 & 1\end{array}\right)$ an orthogonal basis of Hilbert-Schmidt space. Due to the uniqueness of the Hermitian projection operator $\mathcal{P}_{\left\{\hat{M}_{m}\right\}}$ this makes it particularly easy to calculate the projected density operator $\mathcal{P}_{\left\{\hat{M}_{m}\right\}} \hat{\rho}$ representing the information about the density operator $\hat{\rho}$ provided by the measurement. We find (with $\nu=0)$ :

$$
\begin{aligned}
& \Phi=\frac{\pi}{2}, \delta \neq 0: \mathcal{P}_{\left\{\hat{M}_{m}\right\}} \hat{\rho}=\left(\begin{array}{cc}
\rho_{11} & \frac{\rho_{12}-\rho_{21}}{2} \\
-\frac{\rho_{12}-\rho_{21}}{2} & \rho_{22}
\end{array}\right) ; \\
& \Phi \neq \frac{\pi}{2}, \delta=0: \mathcal{P}_{\left\{\hat{M}_{m}\right\}} \hat{\rho}=\left(\begin{array}{cc}
\rho_{11} & \frac{\rho_{12}+\rho_{21}}{2} \\
\frac{\rho_{12}+\rho_{21}}{2} & \rho_{22}
\end{array}\right) ; \\
& \Phi=\frac{\pi}{2}, \delta=0: \mathcal{P}_{\left\{\hat{M}_{m}\right\}} \hat{\rho}=\left(\begin{array}{cc}
\rho_{11} & 0 \\
0 & \rho_{22}
\end{array}\right) .
\end{aligned}
$$

Note that all $\mathcal{P}_{\left\{\hat{M}_{m}\right\}} \hat{\rho}$ are non-negative (cf. Appendix). In the $\Phi=\frac{\pi}{2}, \delta=0$ case only information is obtained on the diagonal elements of $\hat{\rho}$ because for these parameter values the measurement is a non-ideal measurement of PVM $\{|e\rangle\langle e|| g\rangle,\langle g|\}$. It is clear from this that from the informational point of view our parameter choice in section III was not completely appropriate for the purpose of reconstructing the initial density operator. By restricting to $\frac{\pi}{2}$ pulses the measurement cannot retrieve $\operatorname{Im} \rho_{12}$. For a complete determination of $\hat{\rho}$ it is necessary that $\Phi \neq \frac{\pi}{2}$ and $\delta \neq 0$ (keeping $\delta \neq \pm 1$ ).

One remark is in order here. Since the special parameter values $\Phi=\frac{\pi}{2}$ and $\delta=0$ constitute sets of measure zero within the set of all possible values of the parameters, it might be thought that these special values are physically irrelevant because they cannot be attained in practice. In a strict sense this is correct. However, even though in practice the POVMs are complete, this does not mean that the subspace structure is unimportant. As a matter of fact, if the parameter values are near the special values given above, then the quantities $\operatorname{Tr}\left(\hat{\rho}-\hat{\rho}_{\left\{\hat{M}_{m}\left(\Phi=\frac{\pi}{2}, \delta=0\right)\right\}}\right) \hat{M}_{m}$ will be very small. This means that the experimental error in the determination of these quantities is relatively large. Hence, the experimental probabilities will yield relatively poor information about the components of the HilbertSchmidt vector $\hat{\rho}$ orthogonal to $\mathcal{H}_{\left\{\hat{M}_{m}\left(\Phi=\frac{\pi}{2}, \delta=0\right)\right\}}$. Stated differently, sets $\left\{\hat{M}_{m}\right\}$ constituting bases of Hilbert-Schmidt space need not be equivalent from an informational point of view, the quality of the information being largest for an orthogonal basis. A measure of this quality will be discussed elsewhere.

\section{THE HAROCHE-RAMSEY EXPERIMENT AS A JOINT NON-IDEAL MEASUREMENT OF INTERFERENCE AND PATH OBSERVABLES}

Rather than exploiting a second atom, or, equivalently, measuring observable $\left\{\sin ^{2} \Phi \hat{a}^{\dagger} \hat{a}, \cos ^{2} \Phi \hat{a}^{\dagger} \hat{a}\right\}$ of the cavity $C$ field jointly with observable $\{|e\rangle\langle e|| g\rangle,\langle g|\}$, we consider here the field observable $\left\{\hat{M}_{+}, \hat{M}_{-}\right\}$defined by

$$
\hat{M}_{+}=\frac{1}{\pi} \int_{C^{+}} d^{2} \alpha|\alpha\rangle\left\langle\alpha\left|; \hat{M}_{-}=\frac{1}{\pi} \int_{C^{-}} d^{2} \alpha\right| \alpha\right\rangle\langle\alpha|,
$$

$|\alpha\rangle$ a coherent state, and the integrations being over the upper $\left(C^{+}\right)$and lower $\left(C^{-}\right)$complex half-planes, respectively. This observable is a coarsening of the observable $\left\{\frac{1}{\pi}|\alpha\rangle\langle\alpha|\right\}$ measured in the eight-port homodyning detection method [10,30. For $\gamma \rightarrow \infty$ we have $\left\langle\gamma e^{i \Phi}\left|\hat{M}_{m}\right| \gamma e^{i \Phi}\right\rangle \rightarrow \delta_{m+},\left\langle\gamma e^{-i \Phi}\left|\hat{M}_{m}\right| \gamma e^{-i \Phi}\right\rangle \rightarrow \delta_{m-}$. In this limit POVM $\left\{\hat{M}_{+}, \hat{M}_{-}\right\}$is evidently yielding information on the phase shift $\Phi$ caused by the Rb atom, and, hence, is providing path information. In the Haroche-Ramsey experiment [1] the value of $\gamma$ was finite $(\gamma \approx 3)$, causing the distinguishability of the states to be only partial. As will be seen in the following, this loss of path information is compensated by the interference information obtained by measuring a quantity of the $C$ field yielding information on both number and phase. 
In order to interpret the experiment as a measurement in the initial state $\left|\psi_{\text {in }}\right\rangle$ we put

$$
p_{m \pm}=\left\langle\Psi_{f}\left|\left(|m\rangle\langle m| \otimes \hat{M}_{ \pm}\right)\right| \Psi_{f}\right\rangle=\left\langle\psi_{i n}\left|\hat{M}_{m \pm}\right| \psi_{i n}\right\rangle, m=e, g
$$

yielding a POVM $\left\{\hat{M}_{e+}, \hat{M}_{e-}, \hat{M}_{g+}, \hat{M}_{g-}\right\}$ with elements given by

$$
\begin{gathered}
\hat{M}_{e \pm}=\frac{1}{2}\left[\left(1 \pm A-C_{1}\right)\left|S_{1}\right|^{2} \hat{P}_{+}+\left(1 \mp A-C_{1}\right)\left|S_{2}\right|^{2} \hat{P}_{-}+C_{1} \hat{Q}_{e}+C_{2} \hat{S}\right] \\
\hat{M}_{g \pm}=\frac{1}{2}[1 \mp A \delta] \hat{I}-\hat{M}_{e \mp}, \delta=\left|S_{1}\right|^{2}-\left|S_{2}\right|^{2}
\end{gathered}
$$

Here $\left\{\hat{P}_{+}, \hat{P}_{-}\right\}$and $\left\{\hat{Q}_{e}, \hat{Q}_{g}\right\}$ are the path and interference observables (10) and (13) defined above. The constant $A$ is given by

$$
A=\operatorname{erf}(\gamma \sin \Phi)
$$

and $C_{1}$ and $C_{2}$ are given by (18). The operator $\hat{S}$ is defined according to

$$
\hat{S}=i e^{-i \nu \tau} S_{1}^{*} S_{2}\left|p_{+}\right\rangle\left\langle p_{-}\left|-i e^{i \nu \tau} S_{1}^{*} S_{2}\right| p_{-}\right\rangle\left\langle p_{+}\right|,
$$

$\left|p_{ \pm}\right\rangle$being given by $(10)$.

In the phase averaged case the experiment described by this POVM once again is a non-ideal measurement of PVM $\{|e\rangle\langle e|| g\rangle,\langle g|\}$. Restricting to $\nu=\delta=0$ we find

$$
\overline{\hat{M}_{e \pm}}=\frac{1}{4}\left(1-C_{1}\right)|e\rangle\left\langle e\left|+\frac{1}{4}\left(1+C_{1}\right)\right| g\right\rangle\left\langle g\left|, \overline{M_{g \pm}}=\frac{1}{4}\left(1+C_{1}\right)\right| e\right\rangle\left\langle e\left|+\frac{1}{4}\left(1-C_{1}\right)\right| g\right\rangle\langle g|,
$$

yielding for the non-ideality measure the same outcome (19) as obtained in the experiment in which no measurement is performed on the cavity $C$ field. Evidently, in the phase averaged case such a measurement does not improve the information. Indeed, the two measurements are equivalent in the sense defined in [8].

We shall now consider POVM (22) when no phase averaging is performed. We should exclude $\delta= \pm 1$ also here because then also POVM $\left\{\hat{M}_{e \pm}, \hat{M}_{g \pm}\right\}$ reduces to a trivial refinement of PVM $\{|e\rangle\langle e|| g\rangle,\langle g|\}$ (this actually holds true for any choice of the observable of the cavity $C$ field). In the limit $\gamma=0$ the POVM reduces to $\left\{\frac{1}{2} \hat{Q}_{e}, \frac{1}{2} \hat{Q}_{e}, \frac{1}{2} \hat{Q}_{g}, \frac{1}{2} \hat{Q}_{g}\right\}$, representing a trivial refinement of the interference observable $\left\{\hat{Q}_{e}, \hat{Q}_{g}\right\}$. On the other hand, for $\gamma \rightarrow \infty$ the POVM reduces to the trivial refinement $\left\{\left|S_{1}\right|^{2} \hat{P}_{+},\left|S_{2}\right|^{2} \hat{P}_{-},\left|S_{2}\right|^{2} \hat{P}_{+},\left|S_{1}\right|^{2} \hat{P}_{-}\right\}$of the path observable $\left\{\hat{P}_{+}, \hat{P}_{-}\right\}$. We shall now demonstrate that if the $\frac{\pi}{2}$ pulse condition $\delta=0$ is satisfied ( $\nu$ arbitrary) and $\Phi=\frac{\pi}{2}$, the Haroche-Ramsey experiment can be interpreted, in the sense of section IIC, as a joint non-ideal measurement of the incompatible observables $\left\{\hat{Q}_{e}, \hat{Q}_{g}\right\}$ and $\left\{\hat{P}_{+}, \hat{P}_{-}\right\}$. To see this we define the bivariate POVM $\left\{\hat{M}_{m n}\right\}$ :

$$
\left\{\hat{M}_{m n}\right\}=\left(\begin{array}{cc}
\hat{M}_{e+} & \hat{M}_{g+} \\
\hat{M}_{e-} & \hat{M}_{g-}
\end{array}\right)
$$

For the two marginals we find, respectively,

$$
\left(\begin{array}{c}
\hat{M}_{e+}+\hat{M}_{g+} \\
\hat{M}_{e-}+\hat{M}_{g-}
\end{array}\right)=\overbrace{\frac{1}{2}\left(\begin{array}{cc}
1+\operatorname{erf}(\gamma) & 1-\operatorname{erf}(\gamma) \\
1-\operatorname{erf}(\gamma) & 1+\operatorname{erf}(\gamma)
\end{array}\right)}^{\left(\lambda_{m n}\right)}\left(\begin{array}{c}
\hat{P}_{+} \\
\hat{P}_{-}
\end{array}\right)
$$

and

$$
\left(\begin{array}{c}
\hat{M}_{e+}+\hat{M}_{e-} \\
\hat{M}_{g+}+\hat{M}_{g-}
\end{array}\right)=\overbrace{\frac{1}{2}\left(\begin{array}{cc}
1+e^{-2 \gamma^{2}} & 1-e^{-2 \gamma^{2}} \\
1-e^{-2 \gamma^{2}} & 1+e^{-2 \gamma^{2}}
\end{array}\right)}^{\left(\mu_{m n}\right)}\left(\begin{array}{c}
\hat{Q}_{e} \\
\hat{Q}_{g}
\end{array}\right) .
$$

In the limits $\gamma \rightarrow \infty$ and $\gamma=0$ the marginals describe ideal measurements of path and interference, respectively. The non-ideality measures $J_{(\lambda)}$ and $J_{(\mu)}$ corresponding to the non-ideality matrices $\left(\lambda_{m n}\right)$ and $\left(\mu_{m n}\right)$ are found according to 


$$
\begin{aligned}
& J_{(\lambda)}=-\frac{1}{2}\left[(1+\operatorname{erf}(\gamma)) \ln \left(\frac{1+\operatorname{erf}(\gamma)}{2}\right)+(1-\operatorname{erf}(\gamma)) \ln \left(\frac{1-\operatorname{erf}(\gamma)}{2}\right)\right] \\
& J_{(\mu)}=-\frac{1}{2}\left[\left(1+e^{-2 \gamma^{2}}\right) \ln \left(\frac{1+e^{-2 \gamma^{2}}}{2}\right)+\left(1-e^{-2 \gamma^{2}}\right) \ln \left(\frac{1-e^{-2 \gamma^{2}}}{2}\right)\right] .
\end{aligned}
$$

For the special values of the parameters considered here we have $\left|\left\langle p_{i} \mid q_{j}\right\rangle\right|=1 / \sqrt{2}, i= \pm, j=e, g$. Hence, for the observables $\left\{\hat{Q}_{e}, \hat{Q}_{g}\right\}$ and $\left\{\hat{P}_{+}, \hat{P}_{-}\right\}$the right-hand side of inequality (田) is non-vanishing, and it is impossible that both $J_{(\lambda)}$ and $J_{(\mu)}$ are equal to zero. In figure $4 J_{(\lambda)}$ is plotted versus $J_{(\mu)}$ as a function of the parameter $\gamma$. The resulting curve clearly exhibits the idea of complementarity expressed by inequality (4): the experiment constitutes a less accurate measurement of the interference observable as the path observable is determined more accurately by increasing $\gamma$ (and vice versa). It is impossible that both $J_{(\lambda)}$ and $J_{(\mu)}$ simultaneously have small values.

The nice feature of the $\Phi=\frac{\pi}{2}$ condition is that the $\gamma$ dependence of the marginals is completely taken into account by the non-ideality matrices $\left(\lambda_{m n}\right)$ and $\left(\mu_{m n}\right), \operatorname{PVMs}\left\{\hat{Q}_{e}, \hat{Q}_{g}\right\}$ and $\left\{\hat{P}_{+}, \hat{P}_{-}\right\}$being independent of $\gamma$. This feature is partly lost if we allow values $\Phi \neq \frac{\pi}{2}$. For general values of the parameters we can represent POVM (22) in the following way:

$$
\begin{aligned}
& \left.\hat{M}_{e+}=\frac{1}{2}\left[(1+A)\left|S_{1}\right|^{2}\left|p_{+}\right\rangle\left\langle\left. p_{+}|+(1-A)| S_{2}\right|^{2} \mid p_{-}\right\rangle\left\langle p_{-}\left|+\left\{C e^{-i \nu \tau} S_{1}^{*} S_{2}\right\}\right| p_{+}\right\rangle\left\langle p_{-}\right|+\text {h.c. }\right\}\right] \\
& \left.\hat{M}_{e-}=\frac{1}{2}\left[(1-A)\left|S_{1}\right|^{2}\left|p_{+}\right\rangle\left\langle\left. p_{+}|+(1+A)| S_{2}\right|^{2} \mid p_{-}\right\rangle\left\langle p_{-}\left|+\left\{C e^{-i \nu \tau} S_{1}^{*} S_{2}\right\}\right| p_{+}\right\rangle\left\langle p_{-}\right|+\text {h.c. }\right\}\right] \\
& \left.\hat{M}_{g+}=\frac{1}{2}\left[(1+A)\left|S_{2}\right|^{2}\left|p_{+}\right\rangle\left\langle\left. p_{+}|+(1-A)| S_{1}\right|^{2} \mid p_{-}\right\rangle\left\langle p_{-}\left|-\left\{C e^{-i \nu \tau} S_{1}^{*} S_{2}\right\}\right| p_{+}\right\rangle\left\langle p_{-}\right|+\text {h.c. }\right\}\right] \\
& \left.\hat{M}_{g-}=\frac{1}{2}\left[(1-A)\left|S_{2}\right|^{2}\left|p_{+}\right\rangle\left\langle\left. p_{+}|+(1+A)| S_{1}\right|^{2} \mid p_{-}\right\rangle\left\langle p_{-}\left|-\left\{C e^{-i \nu \tau} S_{1}^{*} S_{2}\right\}\right| p_{+}\right\rangle\left\langle p_{-}\right|+\text {h.c. }\right\}\right] .
\end{aligned}
$$

From this we find as one marginal

$$
\left(\begin{array}{c}
\hat{M}_{e+}+\hat{M}_{g+} \\
\hat{M}_{e-}+\hat{M}_{g-}
\end{array}\right)=\overbrace{\frac{1}{2}\left(\begin{array}{cc}
1+A & 1-A \\
1-A & 1+A
\end{array}\right)}^{\left(\lambda_{m n}^{\prime}\right)}\left(\begin{array}{c}
\hat{P}_{+} \\
\hat{P}_{-}
\end{array}\right),
$$

$A=\operatorname{erf}(\gamma \sin \Phi)$, which still is a non-ideal measurement of the the path observable $\left\{\hat{P}_{+}, \hat{P}_{-}\right\}$. However for the other marginal we get

$$
\left(\begin{array}{c}
\hat{M}_{e+}+\hat{M}_{e-} \\
\hat{M}_{g+}+\hat{M}_{g-}
\end{array}\right)=\overbrace{\left(\begin{array}{cc}
\mu & 1-\mu \\
1-\mu & \mu
\end{array}\right)}^{\left(\mu_{m n}^{\prime}\right)}\left(\begin{array}{c}
\hat{Q}_{e}^{\prime} \\
\hat{Q}_{g}^{\prime}
\end{array}\right)
$$

with $\mu=\left(1-\sqrt{1-\left(1-\delta^{2}\right)\left(1-|C|^{2}\right)}\right) / 2$ and $\left\{\hat{Q}_{e}^{\prime}, \hat{Q}_{g}^{\prime}\right\}$ different from $\left\{\hat{Q}_{e}, \hat{Q}_{g}\right\}$. Since PVM $\left\{\hat{Q}_{e}^{\prime}, \hat{Q}_{g}^{\prime}\right\}$ turns out to be dependent on $\gamma$ the experiment no longer is a joint measurement of one stable PVM pair when varying $\gamma$. Nevertheless for each set of parameters PVM $\left\{\hat{Q}_{e}^{\prime}, \hat{Q}_{g}^{\prime}\right\}$ is incompatible with the path observable, and inequality (四) is satisfied by the non-ideality measures $J_{\left(\lambda^{\prime}\right)}$ and $J_{\left(\mu^{\prime}\right)}$. Since the parameters $A$ and $|C|$ depend on $\gamma$ and $\Phi$ only as $\gamma$ sin $\Phi$, this latter quantity, together with $\delta$, is determining the measure of complementarity of observables $\left\{\hat{Q}_{e}^{\prime}, \hat{Q}_{g}^{\prime}\right\}$ and $\left\{\hat{P}_{+}, \hat{P}_{-}\right\}$. By comparing figures 5 and 3 it is seen that, contrary to the Davidovich-Haroche experiment, these observables are complementary for both $\gamma=0$ and $\gamma \rightarrow \infty$, complementarity being largest for $\delta=0$.

The measurement represented by POVM (22) is not a complete one. It can be verified that, if $\gamma \neq 0$, the operator $\hat{T}=i C e^{-i \nu \tau} S_{1}^{*} S_{2}\left|p_{+}\right\rangle\left\langle p_{-}\right|+$h.c. is orthogonal to all operators of the POVM. However, for $\delta \neq \pm 1$ no parameter values exist for which subspace $\mathcal{H}_{\left\{\hat{M}_{m}\right\}}$ has dimension smaller than 3 . This demonstrates the informational superiority of the present measurement, based on homodyning, over the one measuring photon number. By refining the partition $\left(\mathbf{C}^{+}, \mathbf{C}^{-}\right)$of the complex plane POVM (22) can easily be refined to one spanning the whole Hilbert-Schmidt space of $2 \times 2$ matrices, allowing a complete determination of the incoming state $\psi_{\text {in }}$ of the atom. 


\section{SUMMARY AND CONCLUSIONS}

In this paper we studied a number of atomic beam experiments related to the Ramsey experiment. Whereas this latter experiment is a pure interference measurement, in the experiments studied here also 'which-way' information can be obtained. This is achieved by inserting a third microwave cavity, $C$, between the ones already present in the Ramsey experiment, and measuring some observable of the cavity $C$ field after the atom has passed. Three different measurement arrangements were considered. In the first (referred to as the Davidovich-Haroche experiment) a second atom was used as a probe, in the second a measurement of photon number is performed instead. In the third arrangement homodyne optical detection of the cavity $C$ field is contemplated. The experiments were demonstrated to yield new examples of generalized measurements, to be described by positive operator-valued measures. POVMs were calculated explicitly for different values of the experimental parameters.

The experiments are interesting for two reasons. In the first place they can be interpreted as joint non-ideal measurements of incompatible observables, thus clarifying the notion of complementarity. It was found that, although all measurements satisfy inequality (何), only the last experiment exhibits complementarity in the sense that there exist two limiting values of the experimental parameters, for one of which the measurement is a pure interference measurement in which 'which-way' information is maximally disturbed, whereas in the other limit it is a pure 'whichway' measurement in which no interference can be observed. It was demonstrated that in general by the other measurement arrangements this "classical" type of complementarity need not be satisfied. In the second place, comparison of the different measurements can give insight into the question of which information is provided by a (generalized) quantum mechanical measurement. For this purpose the subspaces of Hilbert-Schmidt space, spanned by the operators of the POVM, were determined for different measurement arrangements and different values of the parameters. It was found that a measurement of the second atom in the Davidovich-Haroche experiment is equivalent to a non-ideal measurement of cavity $C$ photon number. Also with respect to measurement of the initial state of the atom this equivalence turns out to hold. In this respect the third arrangement, in which the photon number measurement is replaced by a measurement yielding also phase information, is shown to be superior.

An interesting aspect of the generalized measurements considered here, is that the measured probability distributions dependent on the initial phase of the microwave fields. This makes an experimental realization particularly challenging.

[1] M. Brune, E. Hagley, J. Dreyer, X. Maitre, A. Maali, C. Wunderlich, J. M. Raimond and S. Haroche, Phys. Rev. Let. 77, 4887 (1996).

[2] E. B. Davies, Quantum Theory of Open Systems, Academic Press, London, 1976; A. S. Holevo, Probabilistic and Statistical Aspects of Quantum Theory, North-Holland, Amsterdam, 1982; G. Ludwig, Foundations of Quantum Mechanics, Springer, Berlin, 1983, Vols. I and II; P. Busch, M. Grabowski and P. J. Lahti, Operational quantum mechanics, Springer-Verlag, Berlin, Heidelberg, 1995.

[3] W. M. de Muynck, to be published in Foundations of Physics, e-print archive quant-ph/9901010.

[4] E.P. Storey, S.M. Tan, M.J. Collett and D.F. Walls, Nature 375, 368 (1995).

[5] M.O. Scully, B.-G. Englert and H. Walther, Nature 351, 111 (1991); B.-G. Englert, M.O. Scully and H. Walther, Nature 375, 367 (1995).

[6] S. Duerr, T. Nonn and G. Rempe, Nature 395, 33 (1998).

[7] L.E. Ballentine, Rev. Mod. Phys. 42, 358 (1970).

[8] H. Martens and W. de Muynck, Found. of Phys. 20, 255, 357 (1990).

[9] N.G. Walker and J.E. Caroll, Electr. Lett. 20, 981 (1984).

[10] H.P. Yuen, J.H. Shapiro, IEEE Trans. Inform. Theory IT-26, 78 (1980).

[11] J. Summhammer, H. Rauch, and D. Tuppinger, Phys. Rev. A 36, 4447 (1987).

[12] H. Martens and W.M. de Muynck, Journ. Phys. A: Math. Gen. 26, 2001 (1993).

[13] W.M. de Muynck, W.W. Stoffels, and H. Martens Physica B 175, 127 (1991).

[14] Norman F. Ramsey, Molecular Beams, Oxford at the Clarendon Press, First published 1956, Reprinted lithographically in Great Brittain from corrected sheets of the first edition 1963, 1969.

[15] W.M. de Muynck and H. Martens, Phys. Rev. A 42, 5079 (1990).

[16] D. Leibfried, T. Pfau and C.Monroe, Physics Today, april 1998, p. 22.

[17] K. Banaszek, e-print archive quant-ph/9804050. 
[18] C. D'Helon, G.J. Milburn, Phys. Rev. A 54, R25 (1996).

[19] U. Leonhardt, H. Paul and G.M. d'Ariano, Phys. Rev. A 52,4899 (1995).

[20] K. Vogel and H. Risken, Phys. Rev. A 40, 2847 (1989).

[21] W.M. de Muynck. Journ. Phys. A: Math. Gen. 31, 431 (1998).

[22] L. Davidovich, M. Brune, J. M. Raimond and S. Haroche, Phys. Rev. A 53, 1295 (1996).

[23] R. McEliece, The theory of information and coding, Addison-Wesley, London, 1977.

[24] D. Deutsch, Phys. Rev. Lett. 50, 631 (1983).

[25] M.H. Partovi, Phys. Rev. Lett. 50, 1883 (1983).

[26] K. Kraus, Phys. Rev. D 35, 3070 (1987).

[27] H. Maassen and J.B.M. Uffink, Phys. Rev. Lett. 60, 1103 (1988).

[28] H. Paul, Quant. Opt. 3, 169 (1991).

[29] D. Vitali, P. Tombesi and G.J. Milburn, Phys. Rev. A 57, 4930 (1998).

[30] Y. Lai and H.A. Haus, Quant. Opt. 1, 99 (1989); M. Freyberger and W. Schleich, Phys. Rev. A 47, R30 (1993); U. Leonhardt and H. Paul, Phys. Rev. A 47, R2460 (1993).

\section{ACKNOWLEDGMENT}

The authors thank Maarten Jansen for his contribution to the calculations. 


\section{APPENDIX: POSITIVITY OF $\hat{\rho}_{\left\{\hat{M}_{m}\right\}}$ IN THE TWO-DIMENSIONAL CASE}

In this appendix we prove that on a two-dimensional Hilbert space the operator $\hat{\rho}_{\left\{\hat{M}_{m}\right\}}$, obtained by projecting density operator $\hat{\rho}$ according to $(\mathbb{7})$, is a non-negative operator. We assume that the elements of POVM $\left\{\hat{M}_{m}\right\}$ are linearly independent and consider the non-trivial situation $\left\{\hat{M}_{m}\right\} \neq\{\hat{I}\}$ (if $\left\{\hat{M}_{m}\right\}$ is uninformative, i.e. $\left\{\hat{M}_{m}\right\}=\{\hat{I}\}$, we get $\left.\hat{\rho}_{\left\{\hat{M}_{m}\right\}}=\frac{1}{2} \hat{I}>\hat{O}\right)$. Then the dimension of the subspace $\mathcal{H}_{\left\{\hat{M}_{m}\right\}}$ spanned by the elements of $\left\{\hat{M}_{m}\right\}$ is greater than 1, and it is easy to prove that $\mathcal{H}_{\left\{\hat{M}_{m}\right\}}$ contains a maximal PVM $\left\{\hat{P}_{n}\right\}$. Since the subspace $\mathcal{H}_{\left\{\hat{P}_{n}\right\}}$ is a subspace of $\mathcal{H}_{\left\{\hat{M}_{m}\right\}}$, the orthogonal projections $\mathcal{P}_{\left\{\hat{P}_{n}\right\}}$ and $\mathcal{P}_{\left\{\hat{M}_{m}\right\}}$ satisfy $\mathcal{P}_{\left\{\hat{P}_{n}\right\}} \mathcal{P}_{\left\{\hat{M}_{m}\right\}}=\mathcal{P}_{\left\{\hat{P}_{n}\right\}}$, which implies $\operatorname{Tr} \hat{\rho}_{\left\{\hat{M}_{m}\right\}} \hat{P}_{n}=\operatorname{Tr} \hat{\rho} \hat{P}_{n}$. So in the $\left\{\hat{P}_{n}\right\}$-representation we get

$$
\hat{\rho}=\left(\begin{array}{cc}
p & q \\
q^{*} & 1-p
\end{array}\right) ; \hat{\rho}_{\left\{\hat{M}_{m}\right\}}=\left(\begin{array}{cc}
p & r \\
r^{*} & 1-p
\end{array}\right) .
$$

Because of the fact that $\mathcal{P}_{\left\{\hat{M}_{m}\right\}}$ is an orthogonal projection onto $\mathcal{H}_{\left\{\hat{M}_{m}\right\}}$ we should also have $\operatorname{Tr} \hat{\rho}_{\left\{\hat{M}_{m}\right\}} \hat{\rho}_{\left\{\hat{M}_{m}\right\}}^{\perp}=0$, with $\hat{\rho}_{\left\{\hat{M}_{m}\right\}}^{\perp}=\left(\mathcal{I}-\mathcal{P}_{\left\{\hat{M}_{m}\right\}}\right) \hat{\rho}$, implying $\operatorname{Re}\left(q^{*} r\right)-|r|^{2}=0$, and hence

$$
|q| \geq|r|
$$

Denoting the eigenvalues of $\hat{\rho}_{\left\{\hat{M}_{m}\right\}}$ by $\lambda_{1}$ and $\lambda_{2}$, we find $\lambda_{1} \lambda_{2}=p(1-p)-|r|^{2}$. We already know that $\lambda_{1}+\lambda_{2}=1$. So both eigenvalues are non-negative if

$$
p(1-p)-|r|^{2} \geq 0 .
$$

But since $\hat{\rho}>\hat{O}$ implies $p(1-p)-|q|^{2}>0$ it directly follows from (26) that condition (27) is satisfied. Hence in the two-dimensional case $\hat{\rho}_{\left\{\hat{M}_{m}\right\}}$ is a non-negative operator. 
FIG. 1. The Haroche-Ramsey experiment.

FIG. 2. Comparison of non-idealities $J^{\overline{H R}}$ and $J^{\overline{D H}}$ of the Haroche-Ramsey and Davidovich-Haroche experiments.

FIG. 3. Plots of a) $J_{(\lambda)}$ and b) $J_{(\mu)}$ as functions of $\gamma$ and $\Phi$.

FIG. 4. Plot of $J_{(\lambda)}$ versus $J_{(\mu)}$ for $\delta=0, \Phi=\frac{\pi}{2}$, demonstrating complementarity of interference and path observables. The straight line represents the lower bound of $J_{(\lambda)}+J_{(\mu)}$, given by inequality (仼).

FIG. 5. Plots of a) $J_{\left(\lambda^{\prime}\right)}$ and b) $J_{\left(\mu^{\prime}\right)}$ as functions of $\gamma \sin \Phi$ and $\delta$. 


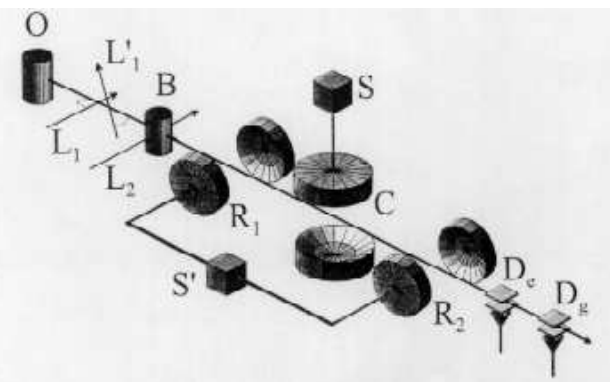

Figure 1

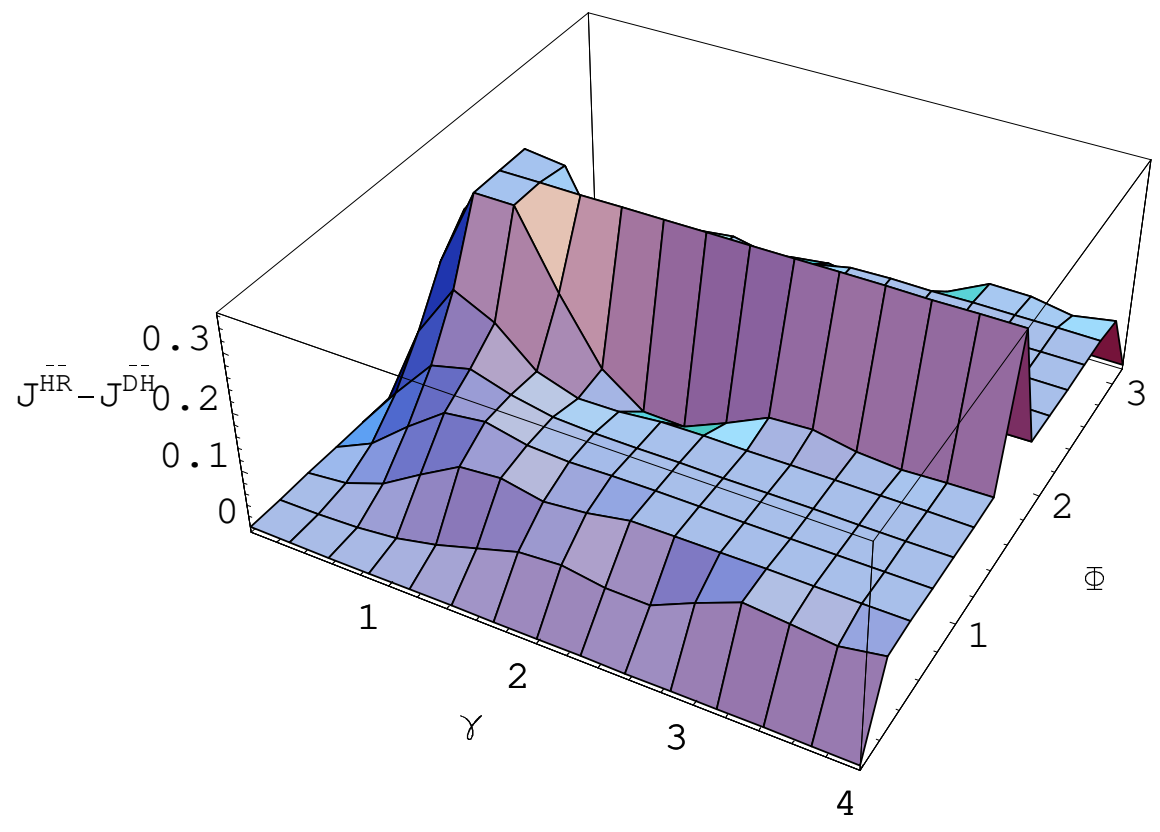

Figure 2 
a)

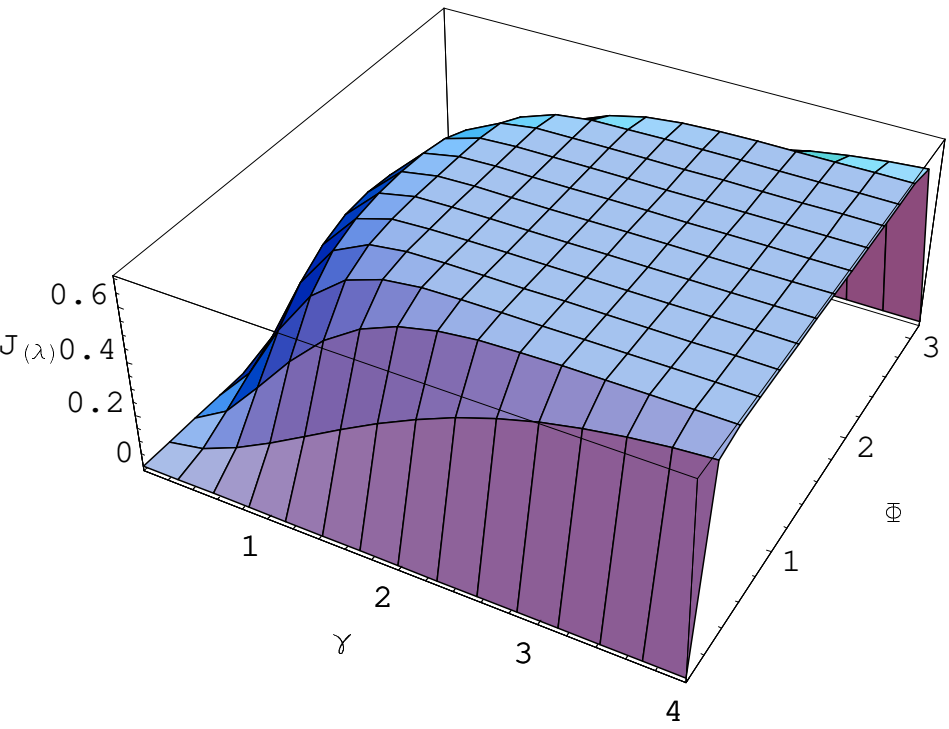

b)

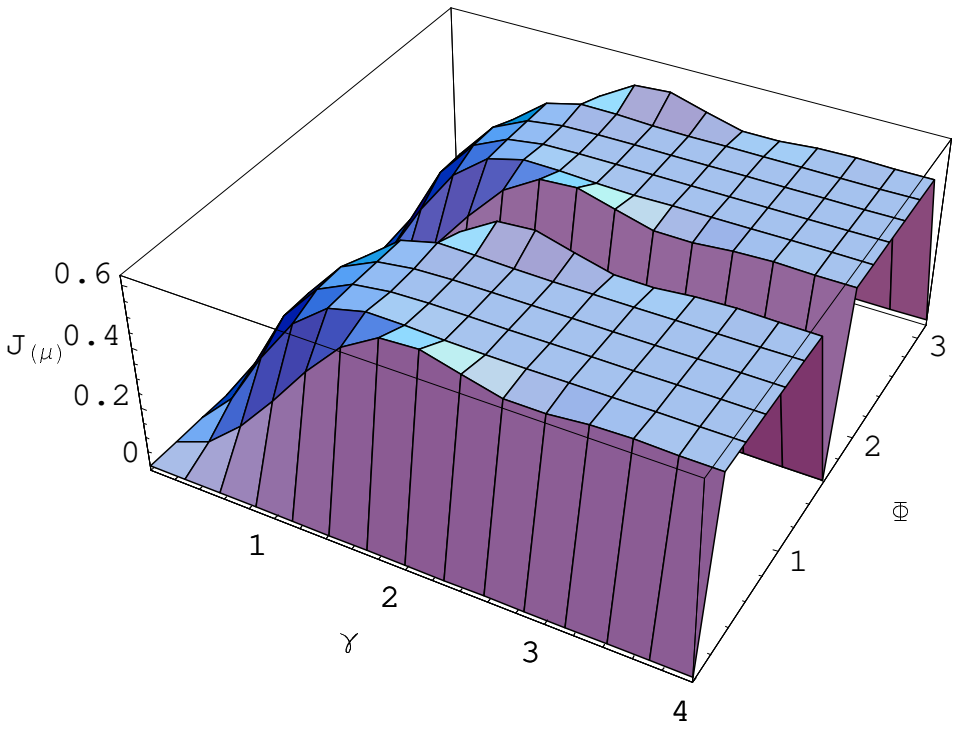

Figure 3 


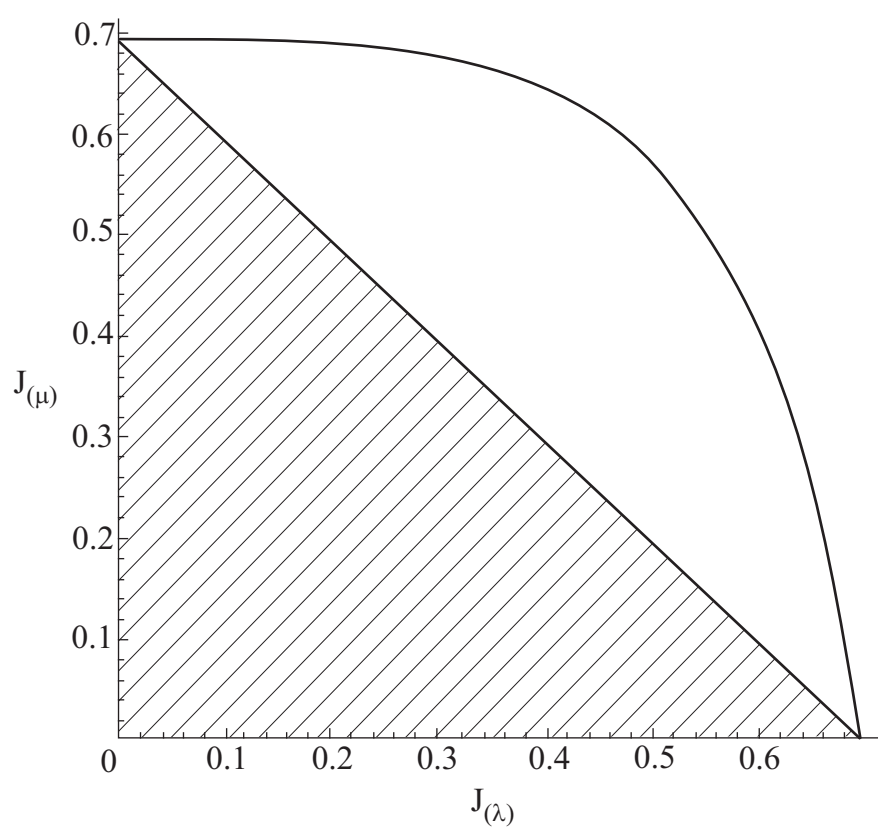

Figure 4. 
a)

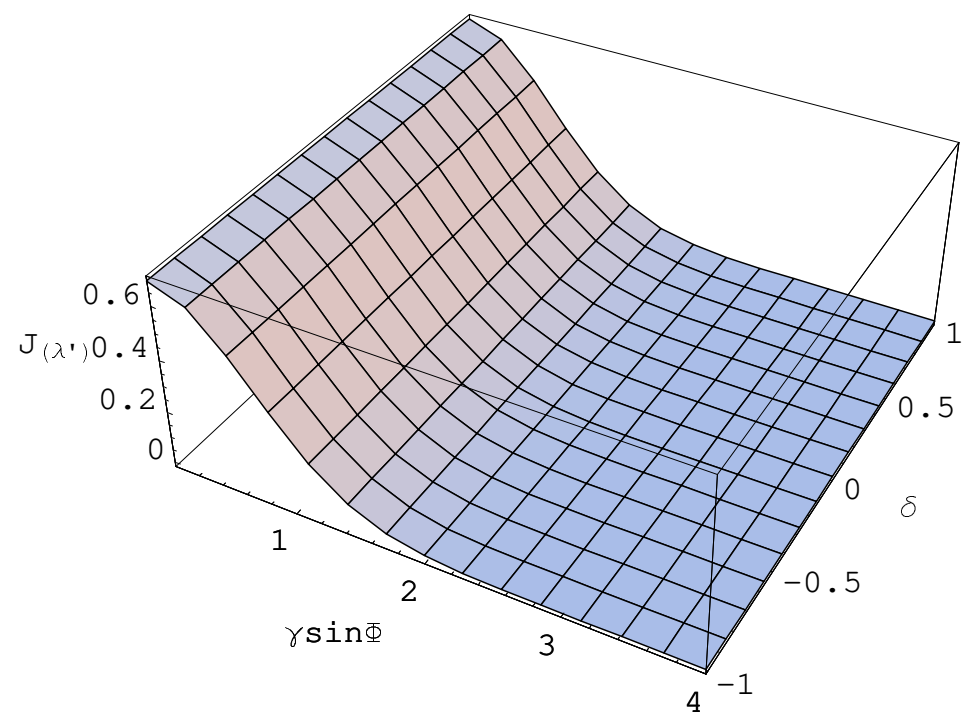

b)

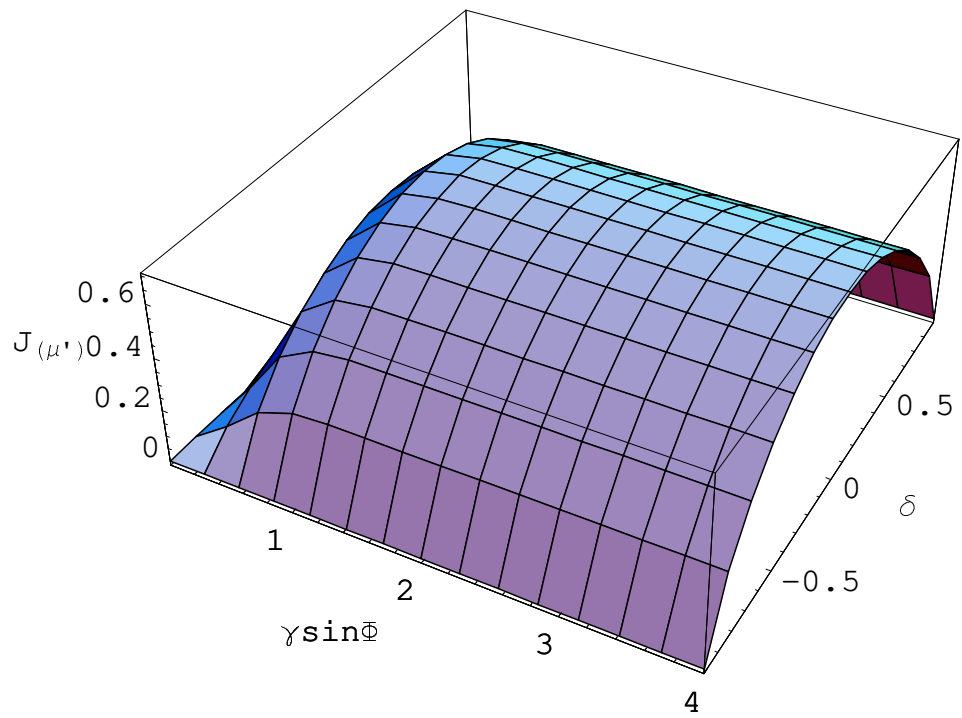

Figure 5 . 